\title{
Reaction mechanisms of fast neutrons on stable Mo isotopes below $21 \mathrm{MeV}$
}

\author{
P. Reimer,${ }^{1,2}$ V. Avrigeanu, ${ }^{3}$ S. V. Chuvaev, ${ }^{4}$ A. A. Filatenkov, ${ }^{4}$ T. Glodariu, ${ }^{3}$ A. Koning, ${ }^{5}$ A. J. M. Plompen,,${ }^{1, *}$ \\ S. M. Qaim, ${ }^{2}$ D. L. Smith, ${ }^{6}$ and H. Weigmann ${ }^{1}$ \\ ${ }^{1}$ European Commission, Joint Research Centre, Institute for Reference Materials and Measurements, Retieseweg, B-2440 Geel, Belgium \\ ${ }^{2}$ Institut für Nuklearchemie, Forschungszentrum Jülich GmbH, D-52425 Jülich, Germany \\ 3 “Horia Hulubei" National Institute for Physics and Nuclear Engineering, P.O. Box MG-6, 76900 Bucharest, Romania \\ ${ }^{4}$ V. G. Khlopin Radium Institute, 2nd Murinski Avenue 28, St. Petersburg 194021, Russia \\ ${ }^{5}$ Nuclear Research and Consultancy Group, P.O. Box 25, NL-1755 ZG Petten, The Netherlands \\ ${ }^{6}$ Nuclear Engineering Division, Argonne National Laboratory, Argonne, Illinois 60439 \\ (Received 31 August 2004; published 29 April 2005)
}

\begin{abstract}
A large number of new measurements with the activation technique were performed for $(n, 2 n)$ and neutron-induced $\Delta Z=1,2$ reaction cross sections on the stable molybdenum isotopes in the energy range from 13.5 to $21 \mathrm{MeV}$. First results were obtained for the ${ }^{92} \mathrm{Mo}(n, 2 n){ }^{91} \mathrm{Mo}^{\mathrm{m}},{ }^{92} \mathrm{Mo}(n, \alpha){ }^{89} \mathrm{Zr}^{\mathrm{m}}$, $\left.{ }^{94} \mathrm{Mo}(n, 2 n){ }^{93} \mathrm{Mo}^{\mathrm{m}},{ }^{95} \mathrm{Mo}(n, p)\right)^{95} \mathrm{Nb}^{\mathrm{m}},{ }^{96} \mathrm{Mo}(n, p){ }^{96} \mathrm{Nb},{ }^{96} \mathrm{Mo}(n, x){ }^{95} \mathrm{Nb}^{\mathrm{m}},{ }^{97} \mathrm{Mo}(n, p){ }^{97} \mathrm{Nb},{ }^{97} \mathrm{Mo}(n, p){ }^{97} \mathrm{Nb}^{\mathrm{m}}$, ${ }^{97} \mathrm{Mo}(n, x){ }^{96} \mathrm{Nb},{ }^{98} \mathrm{Mo}(n, p){ }^{98} \mathrm{Nb}^{\mathrm{m}},{ }^{98} \mathrm{Mo}(n, x){ }^{97} \mathrm{Nb},{ }^{98} \mathrm{Mo}(n, x){ }^{97} \mathrm{Nb}^{\mathrm{m}}$, and ${ }^{100} \mathrm{Mo}(n, \alpha){ }^{97} \mathrm{Zr}$ reactions, above $16 \mathrm{MeV}$. A significant number of high-accuracy $14 \mathrm{MeV}$ measurements were performed which are in good agreement with the measurements above $16 \mathrm{MeV}$ for reactions studied in both energy ranges. The rather complete database for the molybdenum isotopes was analyzed with two different sets of consistent model calculations: a local and a global approach. The global approach (a blind calculation with the TALYS code) provides a good overall description of the dominant reaction channels, although the $(n, \alpha)$ reactions for the heavy isotopes are overpredicted. The local approach (an adjusted calculation with the STAPRE-H code) describes the shapes and magnitudes of the excitation functions well from the reaction thresholds up to $21 \mathrm{MeV}$ using a consistent parameter set, which was optimized based on all experimental information for the nuclei at hand and their immediate neighbors. The agreement between experimental and calculated data is, in general, good both at the maxima and at the tails of the excitation functions, and both for total activation cross sections of a particular channel and for cross sections leading to isomers, showing the viability of the level densities, the optical models, and the $\gamma$ widths. Comparison of the two model calculations with the data indicates the relevance of an appropriate treatment for preequilibrium (PE) $\alpha$-particle emission for the description of the data above $14 \mathrm{MeV}$. Comparison between the model calculations shows largely different PE deuteron emission contributions to the total $(\Delta Z=1, \Delta A=1)$ cross sections with an additional marked difference in energy dependence. This suggests that emission spectra around $20 \mathrm{MeV}$ are required to establish the magnitude of the PE deuteron emission contribution to this process. New $\gamma$-ray strength functions were established by verification against average $(n, \gamma)$ data and were demonstrated to give good agreement with the measured isomer production cross sections.
\end{abstract}

DOI: 10.1103/PhysRevC.71.044617 PACS number(s): 24.10.-i, 24.60.Dr, 25.40.-h, 28.20.-v

\section{INTRODUCTION}

Direct, preequilibrium (PE) and statistical processes should be considered in order to account for reaction channels that are open in fast-neutron interactions in the energy range up to $20 \mathrm{MeV}$. To assess the impact of different model assumptions and determine the optimum parameters that are needed to describe these processes comprehensively, measurements that address the systematics of the dominant reaction channels are essential.

Molybdenum is an excellent structural metal at elevated temperatures. As a consequence, it has a wide potential for use in neutronic applications such as an accelerator-driven system or a controlled nuclear fusion device. However, despite the large amount of data measured in the case of the ${ }^{92} \mathrm{Mo}$ isotope, there are still many discrepancies even between recent measurements. Three evaluations performed rather recently

${ }^{*}$ Corresponding author. Email address: arjan.plompen@cec.eu.int. show differences of up to $\sim 50 \%[1,2]$ and $\sim 65 \%[2,3]$ for the $(n, p)$ and $(n, \alpha)$ reactions, respectively.

The present work concerns additional systematic measurements for neutron-induced reactions on Mo target nuclei from 13 to $21 \mathrm{MeV}$. These measurements are part of a larger measurement campaign to study the systematics of $(n, 2 n)$ and $\Delta Z=1,2$ reaction cross sections, from 14 to $21 \mathrm{MeV}$ [4-7], where relatively few experimental data exist.

The new and existing measured data for the Mo isotopes provide a good basis for the study of the systematics of the dominant reaction channels. Here, two sets of model calculations are compared with the measured data and with each other. For the first, parameters were determined previously by looking for a best overall description of the available data for the entire range from aluminum to bismuth (global approach). For the second, parameter choices were optimized in the present work for the nuclei of immediate relevance of the reactions studied, together with their immediate neighbors (local approach), respecting known $Z$ and $A$ dependences and the available experimental information. 
TABLE I. Isotopic compositions in (\%) of enriched and natural Mo samples.

\begin{tabular}{|c|c|c|c|c|c|c|c|}
\hline Sample & ${ }^{92} \mathrm{Mo}$ & ${ }^{94} \mathrm{Mo}$ & ${ }^{95} \mathrm{Mo}$ & ${ }^{96} \mathrm{Mo}$ & ${ }^{97} \mathrm{Mo}$ & ${ }^{98} \mathrm{Mo}$ & ${ }^{100} \mathrm{Mo}$ \\
\hline \multicolumn{8}{|c|}{ Natural sample ${ }^{a}$} \\
\hline \multicolumn{8}{|c|}{ Enriched samples ${ }^{\mathrm{b}}$} \\
\hline${ }^{92} \mathrm{Mo}$ & $97.37(10)$ & $0.68(5)$ & $0.52(5)$ & $0.37(5)$ & $0.18(5)$ & $0.40(5)$ & $0.50(5)$ \\
\hline${ }^{94}$ Mo & $0.71(5)$ & $92.03(10)$ & $5.18(10)$ & $0.83(5)$ & $0.40(5)$ & $0.67(5)$ & $0.19(5)$ \\
\hline${ }^{95} \mathrm{Mo}$ & \multicolumn{7}{|c|}{ no enriched sample available } \\
\hline${ }^{96} \mathrm{Mo}$ & $0.18(3)$ & $0.20(5)$ & $0.93(5)$ & $96.76(10)$ & $0.96(5)$ & $0.80(5)$ & $0.17(3)$ \\
\hline${ }^{97} \mathrm{Mo}$ & $0.22(5)$ & $0.24(5)$ & $0.59(5)$ & $1.34(5)$ & $94.25(10)$ & $3.07(10)$ & $0.30(5)$ \\
\hline${ }^{98} \mathrm{Mo}$ & $0.32(2)$ & $0.22(2)$ & $0.45(2)$ & $0.59(2)$ & $0.69(2)$ & $97.18(10)$ & $0.55(2)$ \\
\hline${ }^{100} \mathrm{Mo}$ & $0.53(3)$ & $0.18(3)$ & $0.29(3)$ & $0.34(3)$ & $0.28(3)$ & $0.96(5)$ & $97.42(5)$ \\
\hline
\end{tabular}

${ }^{\mathrm{a}}$ Isotopic composition taken from [14].

${ }^{\mathrm{b}}$ Isotopic composition taken from data sheet received from JAERI.

Both calculational approaches describe the physics consistently but with somewhat different models, making for an interesting comparison. Especially at the higher energies considered here, a good description of the excitation functions requires a good description of the preequilibrium contribution. Here, a comparison between the local approach and the global approach is a comparison between the modified geometrydependent hybrid model and the two-component exciton model. Since multistep processes are very important also at intermediate energies, e.g., for the prediction of cross sections needed for development of accelerator-driven systems [8,9], the improvement of both experimental and theoretical PE knowledge in the range $14-21 \mathrm{MeV}$ is of certain interest.

The measurements are described in Section II. The main assumptions and parameter choices for the model calculations are discussed in Section III. The experimental data and their interpretation in terms of the above mentioned models are discussed in detail in Section IV. The conclusions of the work are given in Section V. Preliminary results have been reported elsewhere [10-13].

\section{EXPERIMENTAL METHOD}

Cross sections were determined by the well known activation method. Two different experimental facilities were used to perform the irradiations. The irradiations in the energy range from 16.1 to $20.5 \mathrm{MeV}$ were done at the IRMM $7 \mathrm{MeV}$ Van de Graaff accelerator in Geel, while the measurements between 13.4 and 14.9 MeV were performed at the KRI Neutron Generator NG-400 in St. Petersburg. The methods that were applied closely follow those detailed in Refs. [4,5,12,15,16].

\section{A. Measurements at IRMM}

The experimental methods that were employed here closely follow the description presented in Ref. [5].

\section{Samples and irradiations}

Both natural and enriched materials were used. Natural samples were prepared by either punching discs of $13 \mathrm{~mm}$ diameter from metallic molybdenum foil or by cutting squares of $1 \mathrm{~cm}^{2}$ size from molybdenum sheets. The enriched samples, borrowed from JAERI-Tokai-mura, ${ }^{1}$ were prepared by wrapping about $50 \mathrm{mg}$ of enriched metal in small paper envelopes of $1 \mathrm{~cm}^{2}$ size. The isotopic compositions of the samples are given in Table I. Al, Nb, Fe, and In foils of $13 \mathrm{~mm}$ diameter were attached on both sides of a sample stack and served to monitor neutron flux.

The ${ }^{3} \mathrm{H}(d, n)^{4} \mathrm{He}$ reaction $(Q=17.59 \mathrm{MeV})$ with a solidstate $\mathrm{Ti} / \mathrm{T}$ target $\left(1.923 \mathrm{mg} / \mathrm{cm}^{2}\right.$ thick) on a silver backing (DT neutron field) was utilized for the irradiation experiments. The samples were irradiated in the $0^{\circ}$ direction at $1 \mathrm{~cm}$ distance using deuteron beams of $1,2,3$, and $4 \mathrm{MeV}$.

A long-counter operated in multichannel-scaling acquisition mode was used to record the time profile of the neutron flux during the experiments. Corrections for time-dependent fluctuations were made following Ref. [5].

\section{Mean neutron energy and background fluence}

The neutron energy $E(\theta)$ and yield $Y(\theta)$ at a given nominal angle $\theta$ have been calculated in two ways: The program KINEMA [17] uses the cross-section evaluation of Liskien et al. [18] and the stopping powers of Anderson and Ziegler [19]. The new program ENERGYSET [20] uses the cross-section evaluation of Drosg [21] and the stopping power evaluation of Ziegler [22]. The results of both calculations are well within the uncertainties for the corrections described below.

The mean energy $\bar{E}$ of primary neutrons interacting with a sample is calculated as

$$
\bar{E}=\frac{\int_{0}^{\theta_{\max }} E(\theta) Y(\theta) \theta d \theta}{\int_{0}^{\theta_{\max }} Y(\theta) \theta d \theta},
$$

where $\theta_{\max }$ denotes the maximum angle under which the neutrons strike the sample. For this angle and for $0^{\circ}$, the energy and yield are calculated with the code KINEMA. Based on these values, the angular dependence of neutron energy and yield were approximated with quadratic equations.

$$
E(\theta)=E(0)\left(1+a \theta^{2}\right) \quad \text { and } \quad Y(\theta)=Y(0)\left(1+b \theta^{2}\right),
$$

\footnotetext{
${ }^{1}$ Japanese Atomic Energy Research Institute, Tokai-mura, Japan.
} 
respectively. From this, the two unknowns $a$ and $b$ can easily be derived, and one finally obtains the mean energy:

$$
\bar{E}=E(0) \frac{1+\frac{1}{2}(a+b) \theta_{\max }^{2}+\frac{1}{3} a b \theta_{\max }^{4}}{1+\frac{1}{2} b \theta_{\max }^{2}} .
$$

Here, $E(\Theta)$ is itself a mean neutron energy at the angle $\Theta$, i.e., $E(\Theta)$ is an average over the range of neutron energies that results from the slowing down of deuterons in the $\mathrm{Ti} / \mathrm{T}$ deposit. It was verified that $\bar{E}$ remains unchanged if an average is taken of the angle integrated mean energies at zero and maximum deuteron energy loss. Thus, it was verified that a full integration over the combined distribution versus angle and deuteron slowing down was not required. The effects of both deuteron energy loss in the $\mathrm{Ti} / \mathrm{T}$ deposit and the neutron angular range are included in the estimate for the neutron energy spread.

The well-known monitor reaction ${ }^{27} \mathrm{Al}(n, \alpha){ }^{24} \mathrm{Na}$ [23] was used to determine the neutron fluence for the cross section measurements. In the case of the shorter-lived reaction products, where the irradiation time was too short to allow buildup of enough ${ }^{24} \mathrm{Na}$ activity, the ${ }^{27} \mathrm{Al}(n, p){ }^{27} \mathrm{Mg}$ reaction was used as a reference. After this short irradiation, a second run was performed under the same beam conditions and with the same sample geometry but long enough to allow formation of enough activity from the ${ }^{27} \mathrm{Al}(n, \alpha)^{24} \mathrm{Na}$ reaction. Using the long-counter for normalization, the flux could be extrapolated for the short run. The flux values from both reactions agreed within the uncertainties. The method thus closely follows that described previously in Ref. [5].

The $\left.{ }^{56} \mathrm{Fe}(n, p)\right)^{56} \mathrm{Mn}$ and ${ }^{93} \mathrm{Nb}(n, 2 n){ }^{92} \mathrm{Nb}^{\mathrm{m}}$ reactions were used together with time of flight measurements (TOF) to correct for low energy neutrons originating from the target $([5,24])$. These corrections required excitation curve shape data, taken from experimental data supplemented with STAPRE-H calculations. These excitation curves were folded with flux distributions measured with the TOF technique, under similar but not identical conditions. In particular, the time/irradiation-history dependence of the strength of certain low energy components is significant. Therefore, these flux distributions were subdivided into three groups for which the individual magnitudes in the present conditions were determined using the above mentioned reactions.

The magnitude of the corrections for low-energy neutrons increases with incident deuteron energy and depends on the threshold of the reaction. The correction for $\mathrm{Nb}$ was negligible. For the ${ }^{27} \mathrm{Al}(n, \alpha)^{24} \mathrm{Na}$ reference reaction, a maximum correction of $15 \%$ had to be applied for $4 \mathrm{MeV}$ deuteron energy. The magnitude of this correction increases with the integrated deuteron current at each incident deuteron energy for a given $\mathrm{Ti} / \mathrm{T}$ target.

\section{Measurement of radioactivity}

Standard $\gamma$-ray spectroscopy was employed for the measurement of the radioactivity. Lead-shielded HPGe detectors were used, which were connected to personal computer data acquisition systems via separate analog-to-digital converters (ADCs). The detectors were controlled with the MAESTRO software supplied by EG\&G Ortec, and the S100 system of Canberra. To obtain maximum counting statistics, the monitor foils and samples were placed directly on the detector cap and fixed with adhesive tape. For all detectors, the photo-peak efficiency was determined using calibrated standard sources supplied by PTB, Braunschweig, Germany and by DAMRI, Gif-sur-Yvette, France. An analytical function [25] was used to describe the measured calibration points. Samples were typically placed on the detector, requiring corrections for coincidence summing effects for the ${ }^{93} \mathrm{Mo}^{\mathrm{m}}{ }^{99} \mathrm{Mo},{ }^{96} \mathrm{Nb}$, ${ }^{98} \mathrm{Nb}^{\mathrm{m}}$, and ${ }^{94} \mathrm{Nb}$ activities. For ${ }^{93} \mathrm{Mo}^{\mathrm{m}},{ }^{99} \mathrm{Mo},{ }^{96} \mathrm{Nb}$, and ${ }^{94} \mathrm{Nb}$, it was possible to generate sufficient activity so that summing corrections could be measured by placing such a specially prepared activity once on the detector and once at a large distance ( $>10 \mathrm{~cm}$ ). However, in the case of ${ }^{98} \mathrm{Nb}^{\mathrm{m}}$, a calculated value was applied. For the latter, the total efficiency was determined as well [26]. Further corrections were applied for $\gamma$-ray abundance, $\gamma$-ray self-absorption, and the sample geometry. The decay data that were used were obtained from Ref. [27].

In Table II, the reaction cross sections measured at IRMM are given.

\section{B. Measurements at KRI}

Measurements were carried out at the KRI Neutron Generator NG-400 using the experimental setup that was well tested in many previous measurements. A comprehensive overview is given in Refs. [11,12].

Cross sections were determined by measuring the activity of samples irradiated by neutrons from the ${ }^{3} \mathrm{H}(d, n)^{4} \mathrm{He}$ reaction. The deuteron beam energy was $280 \mathrm{keV}$. Samples were made of metallic molybdenum of natural abundance (see Table I), with a $14 \mathrm{~mm}$ diameter and a thickness of 150-600 $\mu \mathrm{m}$. Molybdenum discs were sandwiched between two niobium or two aluminum foils that were used for neutron fluence determination. Sample assemblies were located around the target at different angles to the deuteron beam. This provided different mean neutron energies in the region of 13.4-14.9 MeV. The neutron energy spectrum was calculated for every sample by taking into account the real geometry of each irradiation, the reaction cross-section evaluation of Drosg [21], and the stopping power evaluation of Anderson and Ziegler [19]. Real beam and target characteristics were also accounted for [15]. Variations in the neutron flux during irradiation were registered by two independent scintillation detectors. Absolute neutron fluences accumulated by the samples were determined using the ${ }^{93} \mathrm{Nb}(n, 2 n){ }^{92} \mathrm{Nb}^{\mathrm{m}}$ and ${ }^{27} \mathrm{Al}(n, \alpha){ }^{24} \mathrm{Na}$ standard cross sections. The ${ }^{27} \mathrm{Al}(n, \alpha)^{24} \mathrm{Na}$ cross sections were taken from Ref. [23]. In our work, we used the experimental values of the ${ }^{93} \mathrm{Nb}(n, 2 n)^{92} \mathrm{Nb}^{\mathrm{m}}$ cross section obtained in Ref. [11] relative to the same standard, because those data show a smoother behavior of the cross section curve. Differences between the ${ }^{93} \mathrm{Nb}(n, 2 n)^{92} \mathrm{Nb}^{\mathrm{m}}$ cross sections of Ref. [11] and the evaluation of Refs. [23,29] are less than the combined errors of measurement and evaluation and do not exceed $1.35 \%$.

Two detectors were used for $\gamma$-ray counting of the irradiated samples. The first was a HPGe detector with a thin beryllium entrance window, and the second was a $\mathrm{Ge}(\mathrm{Li})$ detector. The HPGe detector had a relative efficiency of $24.7 \%$, and the 
TABLE II. Measured activation cross sections, 16-21 MeV. The standard uncertainty is given for the cross sections, whereas the standard spread is presented for the energies. The energy spread combines the effects of straggling of the deuteron in the Ti/T layer and the range of angles subtended by the sample. The contribution to the neutron energy uncertainty due to the deuteron beam is a few keV.

\begin{tabular}{|c|c|c|c|c|c|c|c|}
\hline \multicolumn{2}{|c|}{${ }^{92} \mathrm{Mo}(n, p)^{92} \mathrm{Nb}^{\mathrm{m}}$} & \multicolumn{2}{|c|}{${ }^{92} \operatorname{Mo}(n, \alpha)^{89} \mathrm{Zr}^{\mathrm{m}}$} & \multicolumn{2}{|c|}{${ }^{92} \mathrm{Mo}(n, 2 n)^{91} \mathrm{Mo}^{\mathrm{m}}$} & \multicolumn{2}{|c|}{${ }^{94} \mathrm{Mo}(n, 2 n){ }^{93} \mathrm{Mo}^{\mathrm{m}}$} \\
\hline$E_{n}(\mathrm{MeV})$ & $\sigma(\mathrm{mb})$ & $E_{n}(\mathrm{MeV})$ & $\sigma(\mathrm{mb})$ & $E_{n}(\mathrm{MeV})$ & $\sigma(\mathrm{mb})$ & $E_{n}(\mathrm{MeV})$ & $\sigma(\mathrm{mb})$ \\
\hline $16.2(3)$ & 43.1(29) & $16.1(1)$ & $6.3(7)$ & 16.1(1) & $55(5)$ & $16.2(3)$ & $9.6(8)$ \\
\hline $18.0(1)$ & $34.3(31)$ & $17.8(2)$ & $6.7(10)$ & $17.8(2)$ & $100(21)$ & $18.0(1)$ & $22.0(18)$ \\
\hline $19.3(1)$ & $40.9(55)$ & 19.1(3) & $6.3(10)$ & 19.1(2) & $117(13)$ & $19.3(1)$ & $28.7(24)$ \\
\hline $20.5(1)$ & $39.7(67)$ & $20.5(2)$ & $6.6(25)$ & $20.5(2)$ & $118(21)$ & $20.5(1)$ & $35.9(37)$ \\
\hline \multicolumn{2}{|c|}{${ }^{96} \mathrm{Mo}(n, p){ }^{96} \mathrm{Nb}$} & \multicolumn{2}{|c|}{$\left.{ }^{95} \mathrm{Mo}(n, p)\right)^{95} \mathrm{Nb}^{\mathrm{m}}$} & \multicolumn{2}{|c|}{$\left.{ }^{96} \mathrm{Mo}(n, x)\right)^{95} \mathrm{Nb}^{\mathrm{m}}$} & \multicolumn{2}{|c|}{${ }^{97} \mathrm{Mo}(n, p){ }^{97} \mathrm{Nb}$} \\
\hline$E_{n}(\mathrm{MeV})$ & $\sigma(\mathrm{mb})$ & $E_{n}(\mathrm{MeV})$ & $\sigma(\mathrm{mb})$ & $E_{n}(\mathrm{MeV})$ & $\sigma(\mathrm{mb})$ & $E_{n}(\mathrm{MeV})$ & $\sigma(\mathrm{mb})$ \\
\hline \multirow[t]{2}{*}{$16.22(5)$} & \multirow[t]{2}{*}{ 19.9(18) } & $16.6(3)$ & $5.4(7)$ & \multirow[t]{2}{*}{$16.2(5)$} & \multirow[t]{2}{*}{$3.3(4)$} & $16.23(4)$ & $18.2(13)$ \\
\hline & & $18.0(1)$ & $4.8(9)$ & & & $18.0(1)$ & $19.4(27)$ \\
\hline $19.3(1)$ & $25.1(25)$ & 19.3(1) & $5.0(13)$ & $19.3(1)$ & $12.0(10)$ & $19.3(1)$ & $22.4(29)$ \\
\hline $20.6(1)$ & $25.3(27)$ & $20.5(1)$ & $5.1(9)$ & $20.6(1)$ & $14.2(21)$ & $20.6(1)$ & $19.3(17)$ \\
\hline \multicolumn{2}{|c|}{${ }^{98} \mathrm{Mo}(n, p){ }^{98} \mathrm{Nb}^{\mathrm{m}}$} & \multicolumn{2}{|c|}{$\left.{ }^{97} \mathrm{Mo}(n, p)\right)^{97} \mathrm{Nb}^{\mathrm{m}}$} & \multicolumn{2}{|c|}{${ }^{97} \mathrm{Mo}(n, x){ }^{96} \mathrm{Nb}$} & \multicolumn{2}{|c|}{$\left.{ }^{98} \mathrm{Mo}(n, x)\right)^{97} \mathrm{Nb}^{\mathrm{m}}$} \\
\hline$E_{n}(\mathrm{MeV})$ & $\sigma(\mathrm{mb})$ & $E_{n}(\mathrm{MeV})$ & $\sigma(\mathrm{mb})$ & $E_{n}(\mathrm{MeV})$ & $\sigma(\mathrm{mb})$ & $E_{n}(\mathrm{MeV})$ & $\sigma(\mathrm{mb})$ \\
\hline $16.2(3)$ & $6.9(6)$ & $16.2(1)$ & $5.4(5)$ & $16.23(4)$ & $8.1(7)$ & $16.2(1)$ & $1.9(2)$ \\
\hline $18.0(1)$ & $7.5(12)$ & $17.8(1)$ & $6.4(14)$ & & & $17.8(1)$ & $9.4(19)$ \\
\hline $19.3(1)$ & $9.5(11)$ & $19.2(2)$ & $6.3(10)$ & $19.3(1)$ & $30.6(42)$ & $19.2(2)$ & $14.2(15)$ \\
\hline $20.5(1)$ & $7.2(8)$ & $20.5(2)$ & $6.6(22)$ & $20.6(1)$ & $37.6(41)$ & $20.6(1)$ & $25.5(71)$ \\
\hline \multicolumn{2}{|c|}{${ }^{98} \mathrm{Mo}(n, x){ }^{97} \mathrm{Nb}$} & \multicolumn{2}{|c|}{${ }^{100} \mathrm{Mo}(n, \alpha)^{97} \mathrm{Zr}$} & \multicolumn{2}{|c|}{${ }^{100} \mathrm{Mo}(n, 2 n)^{99} \mathrm{Mo}$} & & \\
\hline$E_{n}(\mathrm{MeV})$ & $\sigma(\mathrm{mb})$ & $E_{n}(\mathrm{MeV})$ & $\sigma(\mathrm{mb})$ & $E_{n}(\mathrm{MeV})$ & $\sigma(\mathrm{mb})$ & & \\
\hline $16.2(1)$ & $2.9(2)$ & $16.2(3)$ & $3.4(2)$ & $16.2(3)$ & $1272(127)$ & & \\
\hline \multirow[t]{3}{*}{$18.0(1)$} & $12.8(17)$ & $18.0(1)$ & $4.6(4)$ & $18.0(1)$ & $980(78)$ & & \\
\hline & & $19.3(1)$ & $5.3(5)$ & $19.3(1)$ & $1018(180)$ & & \\
\hline & & $20.5(1)$ & $5.2(5)$ & $20.5(1)$ & $814(105)$ & & \\
\hline
\end{tabular}

$\mathrm{Ge}(\mathrm{Li})$ detector had a volume of $160 \mathrm{~cm}^{3}$. The energy resolutions of the HPGe and $\mathrm{Ge}(\mathrm{Li})$ detectors were 1.8 and $4.0 \mathrm{keV}$, respectively, at $1332 \mathrm{keV}$. The detectors were enclosed in passive shields. The background count rate was 0.00064 counts/ $\mathrm{s} / \mathrm{keV}$ for the HPGe detector and 0.00019 counts $/ \mathrm{s} / \mathrm{keV}$ for the $\mathrm{Ge}(\mathrm{Li})$ detector at $1300 \mathrm{keV}$.

All observable $\gamma$-ray peaks were revealed and identified in the spectra. The decay data used for cross-section calculations (half-lives, $\gamma$-ray energies, and yields) were obtained from Ref. [28]. Since both the Table of Isotopes and NUDAT are derived from the ENSDF database and appeared within a few years of each other, the decay data used for the KRI and IRMM data are identical, except for insignificantly small differences in the case of ${ }^{89} \mathrm{Zr}^{\mathrm{m}},{ }^{97} \mathrm{Zr},{ }^{97} \mathrm{Nb}$, and ${ }^{91} \mathrm{Mo}^{\mathrm{m}}$. Reaction cross sections presented in Table III are the weighted averages of cross sections obtained for every $\gamma$-ray line related to the reaction.

\section{NUCLEAR MODEL CALCULATIONS}

Two different sets of nuclear-reaction model calculations have been performed that include the direct-interaction, preequilibrium (PE), and statistical Hauser-Feshbach (HF) contributions. The first set of calculations was performed with the recently developed TALYS code $[9,30]$ using systematics originating from global phenomenological analyses. Therefore, these results should be considered predictions made possible by the actual status of knowledge in the field. These calculations will be referred to as global approach or TALYS calculations, interchangeably. The second set of calculations concerns models used previously for the analysis of the target nucleus ${ }^{51} \mathrm{~V}[6,13]$, the isotopes of nickel and ${ }^{59} \mathrm{Co}$ [31] by means of the computer codes DWUCK4 [32], and the updated version of STAPRE-H95 [33]. Here too, a consistent set of parameters is used for each nucleus that is involved in the calculations. However, the choices for these parameters are optimized for each nucleus on the basis of all available experimental information for the nuclei involved and for neighboring nuclei. These calculations will be referred to as STAPRE-H (SH) calculations or as local approach, interchangeably. Finally, a comparison of the results provided by the two different sets between each other and with the experimental data may describe the corresponding predictive power of "blind" calculations.

\section{A. Global approach}

The global approach applied here to the Mo isotopes is similar to that reported in Ref. [31] for $\mathrm{Ni}$ isotopes 
TABLE III. Measured activation cross sections (mb) for Mo isotopes between 13.5 and 14.8 MeV. Mean and full widths at half maximum (fwhm) of the neutron energy distribution are shown. The uncertainty for the mean energy is $10 \mathrm{keV}$.

\begin{tabular}{|c|c|c|c|c|c|c|c|c|}
\hline \multirow[t]{3}{*}{ Reaction } & \multicolumn{8}{|c|}{ Neutron energy (1st row) and fwhm (2nd row) (MeV) } \\
\hline & 13.48 & 13.64 & 13.87 & 14.05 & 14.28 & 14.45 & 14.64 & 14.82 \\
\hline & 0.29 & 0.28 & 0.28 & 0.29 & 0.31 & 0.34 & 0.39 & 0.45 \\
\hline${ }^{92} \mathrm{Mo}(n, 2 n){ }^{91} \mathrm{Mo}^{\mathrm{m}}$ & & $1.44(60)$ & $3.67(60)$ & $6.44(80)$ & $11.1(13)$ & $16.3(22)$ & $22.6(28)$ & $27.1(32)$ \\
\hline${ }^{92} \mathrm{Mo}(n, x)^{91} \mathrm{Nb}^{\mathrm{m}}$ & 207(18) & $228(19)$ & $235(19)$ & $249(20)$ & $259(20)$ & $250(20)$ & $257(21)$ & $252(22)$ \\
\hline${ }^{92} \mathrm{Mo}(n, x){ }^{91} \mathrm{Nb}$ & $1005(400)$ & $1279(400)$ & $1044(400)$ & $978(400)$ & $1259(400)$ & $1147(400)$ & $832(400)$ & $1008(400)$ \\
\hline${ }^{92} \mathrm{Mo}(n, p)^{92} \mathrm{Nb}^{\mathrm{m}}$ & $79.7(32)$ & $79.3(33)$ & $74.5(33)$ & $73.7(30)$ & $69.3(30)$ & $65.3(24)$ & $63.6(28)$ & $60.4(21)$ \\
\hline${ }^{92} \operatorname{Mo}(n, \alpha){ }^{89} \mathrm{Zr}^{\mathrm{m}}$ & $6.44(27)$ & $6.12(39)$ & $6.65(31)$ & $6.61(40)$ & $6.61(42)$ & $7.20(59)$ & $6.87(30)$ & $6.78(56)$ \\
\hline${ }^{92} \mathrm{Mo}(n, \alpha)^{89} \mathrm{Zr}$ & $21.2(13)$ & $22.4(9)$ & $22.8(9)$ & $23.8(9)$ & $24.4(9)$ & $25.2(10)$ & $25.9(10)$ & $26.7(14)$ \\
\hline${ }^{94} \mathrm{Mo}(n, 2 n){ }^{93} \mathrm{Mo}^{\mathrm{m}}$ & & $1.58(15)$ & $2.21(10)$ & $2.96(29)$ & $3.92(35)$ & $4.63(42)$ & $5.89(60)$ & \\
\hline${ }^{95} \mathrm{Mo}(n, p){ }^{95} \mathrm{Nb}^{\mathrm{ma}}$ & $8.20(78)$ & $7.53(74)$ & $8.04(77)$ & $8.12(80)$ & $8.85(94)$ & $8.72(77)$ & $9.90(87)$ & $9.29(77)$ \\
\hline${ }^{95} \mathrm{Mo}(n, p){ }^{95} \mathrm{Nb}^{\mathrm{b}}$ & $34.5(14)$ & $36.0(14)$ & $36.8(15)$ & $39.0(16)$ & $39.9(16)$ & $42.0(17)$ & $41.4(17)$ & $42.6(17)$ \\
\hline${ }^{96} \mathrm{Mo}(n, p){ }^{96} \mathrm{Nb}^{\mathrm{c}}$ & $18.5(8)$ & $17.9(8)$ & $19.1(8)$ & 20.6(9) & $21.4(9)$ & $22.3(9)$ & $24.5(9)$ & $23.0(9)$ \\
\hline${ }^{97} \mathrm{Mo}(n, p){ }^{97} \mathrm{Nb}^{\mathrm{d}}$ & $14.9(18)$ & $15.0(27)$ & $15.7(24)$ & $16.9(7)$ & $17.8(12)$ & $18.2(20)$ & $19.5(10)$ & $21(11)$ \\
\hline${ }^{98} \mathrm{Mo}(n, p){ }^{98} \mathrm{Nb}^{\mathrm{m}}$ & $3.5(5)$ & $4.0(6)$ & $4.09(36)$ & $4.17(23)$ & $4.40(38)$ & $4.79(51)$ & $4.58(29)$ & $4.80(42)$ \\
\hline${ }^{98} \mathrm{Mo}(n, \alpha){ }^{95} \mathrm{Zr}$ & $4.99(30)$ & $5.05(35)$ & $5.25(45)$ & $5.93(32)$ & $5.93(45)$ & $6.60(36)$ & $6.38(31)$ & $6.66(33)$ \\
\hline${ }^{100} \mathrm{Mo}(n, 2 n){ }^{99} \mathrm{Mo}$ & $1496(60)$ & $1424(57)$ & $1419(53)$ & $1462(59)$ & $1454(54)$ & $1456(57)$ & $1434(54)$ & $1472(53)$ \\
\hline${ }^{100} \operatorname{Mo}(n, \alpha)^{97} \mathrm{Zr}$ & & $2.24(12)$ & & & $2.57(15)$ & $2.80(16)$ & & \\
\hline
\end{tabular}

${ }^{\mathrm{a}}$ Uncorrected for the contribution of the ${ }^{96} \mathrm{Mo}(n, x)^{95} \mathrm{Nb}^{\mathrm{m}}$ reaction.

${ }^{\mathrm{b}}$ Uncorrected for the contribution of the ${ }^{96} \mathrm{Mo}(n, x)^{95} \mathrm{Nb}$ reaction.

${ }^{\mathrm{c}}$ Uncorrected for the contribution of the ${ }^{97} \mathrm{Mo}(n, x){ }^{96} \mathrm{Nb}$ reaction.

${ }^{\mathrm{d}}$ Uncorrected for the contribution of the ${ }^{98} \mathrm{Mo}(n, x){ }^{97} \mathrm{Nb}$ reaction.

and ${ }^{59} \mathrm{Co}$. However, a new version $(0.59)$ of the TALYS code [30] was used, although the description given in Ref. [31] regarding the choices for the optical model [34,35], the direct reaction (distorted-wave Born approximation, DWBA, using ECIS97 [36]), the preequilibrium contributions with the two-component exciton model using Kalbach systematics [37], the level density model [38,39], and the $\gamma$ widths can be almost completely repeated verbatim for the calculations that were performed in this work. The main difference here from the work reported in Ref. [31] is that new parameters for the matrix element of the internal transition rates of the exciton model have been developed [40], and a modified expression for the shell-dependent total level density parameter has been used. Also, a more modern implementation of Kalbach's complex particle emission model has been used, improving the description of $(n, d)$ up to $(n, \alpha)$ channels. As the level density model is of crucial importance, for comparison, the parameters governing the level density model are presented in Table IV.

The results given in this paper represent completely blind predictions. The results for Mo form in fact a subset of an automatically generated database that ranges from ${ }^{27} \mathrm{Al}$ to ${ }^{209} \mathrm{Bi}$. All nuclear structure parameters were independently chosen and all nuclear model parameters are taken from global systematic expressions. This means that for the TALYS results presented in this paper, no particular attention was paid to local level density parameters, preequilibrium parameters, etc., and equally important in the case of isomer cross sections, discrete level branching ratios and spin assignments. We note that TALYS is perfectly able to do a local analysis of reaction data, with adjusted model parameters, but for the present work it was more interesting to assess its global predictive power.
Thus, an interesting comparison can be made between a global approach that reflects the state of knowledge of nuclear model parameters within a certain set of model choices, on the one hand, and on the other, a local approach in which a consistent calculation is made with a somewhat different model that uses adjusted model parameters valid for a small range of mass and charge numbers. Important modeling differences with the local approach, given below, concern the level density, and in particular for complex particle emission, the optical model and the preequilibrium treatment.

\section{B. Local approach}

\section{Nuclear level density parameters}

The nuclear level density (NLD) approach adopted recently $[6,42]$ has also been used in the present work. Table $\mathrm{V}$ shows the maximum number $N_{d}$ and corresponding excitation energy $E_{d}$ of low-lying discrete levels [27] used in the HF calculations. Also shown are the values for $N_{d}$ and $E_{d}$ that were used together with the average $s$-wave nucleon resonance spacings $D_{0}$ to derive the level-density parameter $a$ and the back-shift $\Delta$ of the back-shifted Fermi gas (BSFG) model [43]. The fitted $N_{d}$ value and the calculated $D_{0}$ value show the changes from the recommended values that are required to obtain good agreement for the reaction cross sections. The calculated $D_{0}$ always agrees with the experimental $D_{0}$ value within one standard deviation. In the case of residual nuclei with excited isomeric states, also given is the number of low-lying levels for which the $\gamma$-ray decay scheme is not known experimentally but has a large effect on the calculated isomeric-state cross sections. 
TABLE IV. Level-density parameters applicable to the global approach (TALYS-0.59 calculations). $N_{d}$ is the number of discrete levels used. The levels between $N_{\text {low }}$ and $N_{\text {top }}$ are used to determine the parameters $E_{0}$ and $T$ (temperature) for the Gilbert and Cameron constant temperature model. $P$ is the pairing energy. $E_{m}$ is the energy at which the constant temperature model is matched to the Ignatyuk model. For that model, $\tilde{a}$ is the asymptotic level-density parameter, $\gamma$ is the damping constant, and $E_{\mathrm{sh}}$ is the shell correction energy. The resulting level-density parameter $a_{n}$ and $s$ wave level spacing $D_{0}^{\text {cal }}$ at the binding energy are shown as well, and the latter is compared to the experimental values $D_{0}^{\exp }$, which are taken from [41].

\begin{tabular}{|c|c|c|c|c|c|c|c|c|c|c|c|c|c|}
\hline Nucleus & $N_{\text {low }}$ & $N_{\text {top }}$ & $\begin{array}{c}N_{d} \\
(\mathrm{MeV})\end{array}$ & $\begin{array}{c}E_{0} \\
(\mathrm{MeV})\end{array}$ & $\begin{array}{c}T \\
(\mathrm{MeV})\end{array}$ & $\begin{array}{c}P \\
(\mathrm{MeV})\end{array}$ & $\begin{array}{c}E_{m} \\
(\mathrm{MeV})\end{array}$ & $\begin{array}{c}\tilde{a} \\
(\mathrm{MeV})^{-1}\end{array}$ & $\begin{array}{c}\gamma \\
(\mathrm{MeV})^{-1}\end{array}$ & $\begin{array}{c}E_{\mathrm{sh}} \\
(\mathrm{MeV})\end{array}$ & $\begin{array}{c}a_{n} \\
(\mathrm{MeV})^{-1}\end{array}$ & $\begin{array}{l}D_{0}^{\exp } \\
(\mathrm{eV})\end{array}$ & $\begin{array}{l}D_{0}^{\text {cal }} \\
(\mathrm{eV})\end{array}$ \\
\hline${ }^{101} \mathrm{Mo}$ & 2 & 22 & 10 & -2.08 & 0.75 & 1.19 & 6.89 & 12.32 & 0.099 & 4.67 & 16.97 & $400 \pm 80$ & 550 \\
\hline${ }^{100} \mathrm{Mo}$ & 2 & 27 & 20 & 0.13 & 0.69 & 2.40 & 7.03 & 12.22 & 0.099 & 3.83 & 15.73 & & 220 \\
\hline${ }^{99} \mathrm{Mo}$ & 2 & 30 & 10 & -1.40 & 0.74 & 1.21 & 6.38 & 12.11 & 0.099 & 3.38 & 15.36 & $970 \pm 200$ & 633 \\
\hline${ }^{98} \mathrm{Mo}$ & 2 & 30 & 10 & -0.06 & 0.77 & 2.42 & 7.67 & 12.01 & 0.100 & 2.45 & 14.20 & $32 \pm 3$ & 343 \\
\hline${ }^{97} \mathrm{Mo}$ & 2 & 30 & 10 & -1.35 & 0.81 & 1.22 & 6.83 & 11.91 & 0.100 & 1.70 & 13.45 & $850 \pm 45$ & 237 \\
\hline${ }^{96} \mathrm{Mo}$ & 2 & 10 & 10 & 0.31 & 0.80 & 2.45 & 7.66 & 11.80 & 0.100 & 1.02 & 12.69 & $55 \pm 8$ & 494 \\
\hline${ }^{95} \mathrm{Mo}$ & 2 & 10 & 10 & -1.20 & 0.88 & 1.23 & 7.33 & 11.70 & 0.101 & 0.10 & 11.78 & $975 \pm 260$ & 352 \\
\hline${ }^{94} \mathrm{Mo}$ & 2 & 14 & 10 & -0.08 & 0.93 & 2.48 & 9.29 & 11.59 & 0.101 & -0.71 & 11.00 & & 921 \\
\hline${ }^{93} \mathrm{Mo}$ & 2 & 30 & 10 & -0.86 & 0.96 & 1.24 & 8.13 & 11.49 & 0.101 & -1.84 & 9.94 & $2100 \pm 300$ & 623 \\
\hline${ }^{92} \mathrm{Mo}$ & 2 & 11 & 10 & 0.82 & 0.98 & 2.50 & 9.33 & 11.39 & 0.102 & -2.67 & 9.46 & & 252 \\
\hline${ }^{91} \mathrm{Mo}$ & 2 & 11 & 10 & -0.34 & 0.91 & 1.26 & 6.91 & 11.28 & 0.102 & -1.58 & 10.08 & & 85 \\
\hline${ }^{90} \mathrm{Mo}$ & 2 & 9 & 10 & 0.09 & 0.97 & 2.53 & 9.53 & 11.18 & 0.102 & -1.13 & 10.39 & & 68 \\
\hline${ }^{100} \mathrm{Nb}$ & 2 & 10 & 10 & -0.92 & 0.52 & 0.00 & 2.61 & 12.22 & 0.099 & 4.70 & 16.56 & & 65 \\
\hline${ }^{99} \mathrm{Nb}$ & 2 & 7 & 5 & -0.66 & 0.63 & 1.21 & 5.17 & 12.11 & 0.099 & 4.58 & 16.33 & & 41 \\
\hline${ }^{98} \mathrm{Nb}$ & 2 & 5 & 5 & -0.34 & 0.59 & 0.00 & 0.26 & 12.01 & 0.100 & 3.15 & 14.84 & & 109 \\
\hline${ }^{97} \mathrm{Nb}$ & 2 & 12 & 10 & 0.26 & 0.60 & 1.22 & 4.14 & 11.91 & 0.100 & 2.74 & 14.27 & & 30 \\
\hline${ }^{96} \mathrm{Nb}$ & 2 & 11 & 10 & -0.10 & 0.51 & 0.00 & 0.35 & 11.80 & 0.100 & 1.86 & 13.40 & & 62 \\
\hline${ }^{95} \mathrm{Nb}$ & 2 & 10 & 10 & -0.59 & 0.74 & 1.23 & 5.74 & 11.70 & 0.101 & 1.72 & 13.14 & & 39 \\
\hline${ }^{94} \mathrm{Nb}$ & 2 & 10 & 10 & -2.06 & 0.82 & 0.00 & 5.27 & 11.59 & 0.101 & 0.59 & 12.08 & $44 \pm 4$ & 108 \\
\hline${ }^{93} \mathrm{Nb}$ & 2 & 24 & 10 & -1.81 & 0.94 & 1.24 & 8.36 & 11.49 & 0.101 & 0.11 & 11.58 & & 85 \\
\hline${ }^{92} \mathrm{Nb}$ & 2 & 11 & 10 & -0.53 & 0.75 & 0.00 & 3.14 & 11.39 & 0.102 & -1.40 & 10.27 & & 302 \\
\hline${ }^{91} \mathrm{Nb}$ & 2 & 23 & 10 & -0.86 & 0.98 & 1.26 & 8.30 & 11.28 & 0.102 & -1.94 & 9.93 & & 19 \\
\hline${ }^{90} \mathrm{Nb}$ & 2 & 15 & 10 & -1.36 & 0.85 & 0.00 & 4.82 & 11.18 & 0.102 & -0.99 & 10.47 & & 40 \\
\hline${ }^{99} \mathrm{Zr}$ & 2 & 24 & 10 & -0.99 & 0.66 & 1.21 & 5.58 & 12.11 & 0.099 & 4.74 & 16.96 & & 2172 \\
\hline${ }^{98} \mathrm{Zr}$ & 2 & 9 & 5 & 0.54 & 0.66 & 2.42 & 6.52 & 12.01 & 0.100 & 3.96 & 15.92 & & 2810 \\
\hline${ }^{97} \mathrm{Zr}$ & 2 & 12 & 10 & -0.43 & 0.69 & 1.22 & 5.26 & 11.91 & 0.100 & 2.50 & 14.32 & $4500 \pm 1000$ & 1723 \\
\hline${ }^{96} \mathrm{Zr}$ & 2 & 28 & 10 & 1.41 & 0.63 & 2.45 & 5.61 & 11.80 & 0.100 & 2.15 & 13.76 & & 1153 \\
\hline${ }^{95} \mathrm{Zr}$ & 2 & 18 & 10 & 0.57 & 0.61 & 1.23 & 3.84 & 11.70 & 0.101 & 1.51 & 13.08 & $3600 \pm 800$ & 455 \\
\hline${ }^{94} \mathrm{Zr}$ & 2 & 10 & 10 & 0.79 & 0.75 & 2.48 & 6.86 & 11.59 & 0.101 & 1.42 & 12.85 & $160 \pm 15$ & 1271 \\
\hline${ }^{93} \mathrm{Zr}$ & 2 & 11 & 10 & -0.58 & 0.81 & 1.24 & 6.18 & 11.49 & 0.101 & 0.48 & 11.92 & $2600 \pm 700$ & 652 \\
\hline${ }^{92} \mathrm{Zr}$ & 2 & 11 & 10 & 0.60 & 0.85 & 2.50 & 7.80 & 11.39 & 0.102 & -0.00 & 11.38 & $570 \pm 100$ & 2021 \\
\hline${ }^{91} \mathrm{Zr}$ & 2 & 15 & 10 & 0.15 & 0.83 & 1.26 & 5.66 & 11.28 & 0.102 & -1.23 & 10.22 & $6400 \pm 1100$ & 1186 \\
\hline${ }^{90} \mathrm{Zr}$ & 2 & 6 & 10 & 1.48 & 0.87 & 2.53 & 7.27 & 11.18 & 0.102 & -1.95 & 9.74 & & 338 \\
\hline${ }^{89} \mathrm{Zr}$ & 2 & 20 & 10 & -0.31 & 0.86 & 1.27 & 6.32 & 11.07 & 0.103 & -0.59 & 10.61 & & 112 \\
\hline${ }^{88} \mathrm{Zr}$ & 2 & 4 & 10 & 1.05 & 0.82 & 2.56 & 7.18 & 10.97 & 0.103 & 0.07 & 11.01 & & 82 \\
\hline
\end{tabular}

\section{Neutron optical model and deformation parameters}

The calculation of the neutron transmission coefficients was carried out by using local optical model (OM) parameter sets validated through the method of Ref. [45], i.e., simultaneous fits of resonance data and broad energy averages over $200 \mathrm{keV}$ of the neutron total cross section for energies up to $20 \mathrm{MeV}$ [46]. We found that the spherical OM of Lagrange [47] provides results similar to those of the recent and more complex vibrational and dispersive models of Smith [48]. The former potential was shown [49] to describe well the differential elastic scattering cross sections of ${ }^{92,100} \mathrm{Mo}$ at neutron energies of 7,9 , and $11 \mathrm{MeV}$, as well as the inelastic scattering cross sections on low-lying excited levels of the isotopes $92,96,98,100$ Mo. Since the opti- cal model potential (OMP) of Lagrange provides overestimated total neutron cross sections at energies below $1 \mathrm{MeV}$ $[47,49]$, it was adopted only above this neutron energy. At the lower energies, we found the predictions of the spherical OMP parameter set obtained by Smith for natural molybdenum [48] to be in better agreement with the experimental total neutron cross sections for ${ }^{92,94,96,98,100} \mathrm{Mo}$, and therefore they were used for all these isotopes, below $1 \mathrm{MeV}$.

Neutron inelastic scattering is the only significant direct interaction which has been taken into account. The distortedwave Born approximation (DWBA) has been used to describe neutron direct inelastic scattering on the discrete excited levels of the target nucleus, by means of the computer code DWUCK4 and the collective state parameters of Kalbach [50]. 
TABLE V. Discrete level number $N_{d}$ up to excitation energy $E_{d}$ [27], as well as the number $N_{b}^{u}$ of low-lying levels for which the $\gamma$-ray decay scheme is not known experimentally, as used in Hauser-Feshbach calculations, and the low-lying levels and $s$-wave nucleon-resonance spacings $D_{0}^{\text {exp }}$ in the nucleon energy range $\Delta E$ above the respective binding energy $B$, for the target-nucleus ground-state spin $I_{0}$, which were fitted in order to obtain the BSFG level-density parameter $a$ and ground-state shift $\Delta$ (corresponding to a spin cutoff factor calculated with a moment of inertia varying linearly from 50 to $75 \%$ of the rigid-body value, for the excitation energies from g.s. to the nucleon binding energy with a reduced radius $\left.r_{0}=1.25 \mathrm{fm}\right)$.

\begin{tabular}{|c|c|c|c|c|c|c|c|c|c|c|c|}
\hline \multirow[t]{2}{*}{ Nucleus } & \multirow[t]{2}{*}{$N_{d}$} & \multirow[t]{2}{*}{$N_{b}^{u}$} & \multirow{2}{*}{$\begin{array}{c}E_{d} \\
(\mathrm{MeV})\end{array}$} & \multicolumn{6}{|c|}{ Fitted level and resonance data } & \multirow{2}{*}{$\begin{array}{c}a \\
(\mathrm{MeV})^{-1}\end{array}$} & \multirow{2}{*}{$\begin{array}{c}\Delta \\
(\mathrm{MeV})\end{array}$} \\
\hline & & & & $N_{d}$ & $\begin{array}{c}E_{d} \\
(\mathrm{MeV})\end{array}$ & $\begin{array}{l}B+\frac{\Delta E}{2} \\
(\mathrm{MeV})\end{array}$ & $I_{0}$ & $\begin{array}{c}D_{0}^{\exp } \\
(\mathrm{eV})\end{array}$ & $\begin{array}{c}D_{0}^{\text {cal }} \\
(\mathrm{eV})\end{array}$ & & \\
\hline${ }^{101} \mathrm{Mo}$ & 23 & & 0.626 & 23 & 0.63 & 5.411 & 0 & $\begin{array}{r}800 \pm 150^{\mathrm{a}} \\
1000 \pm 300^{\mathrm{b}}\end{array}$ & 868 & 12.55 & -1.30 \\
\hline${ }^{100} \mathrm{Mo}$ & 21 & & 2.200 & 30 & 2.46 & & & & & 11.80 & 0.36 \\
\hline${ }^{99} \mathrm{Mo}$ & 41 & & 1.405 & 41 & 1.41 & 5.941 & 0 & $\begin{aligned} 1000 & \pm 200^{\mathrm{a}} \\
941 & \pm 215^{\mathrm{b}}\end{aligned}$ & 1000 & 12.36 & -0.73 \\
\hline${ }^{98} \mathrm{Mo}$ & 23 & & 2.420 & 37 & 2.68 & 8.644 & $5 / 2$ & $\begin{array}{l}75 \pm 20^{\mathrm{a}} \\
37 \pm 2^{\mathrm{b}}\end{array}$ & 77.6 & 11.65 & 0.50 \\
\hline${ }^{97} \mathrm{Mo}$ & 30 & & 1.566 & 30 & 1.57 & 6.831 & 0 & $\begin{array}{r}1050 \pm 200^{\mathrm{a}} \\
876 \pm 110^{\mathrm{b}}\end{array}$ & 955 & 11.40 & -0.60 \\
\hline${ }^{96} \mathrm{Mo}$ & 33 & & 2.875 & 33 & 2.88 & 9.156 & $5 / 2$ & $\begin{array}{c}105 \pm 10^{\mathrm{a}} \\
93 \pm 7^{\mathrm{b}}\end{array}$ & 115 & 10.69 & 0.54 \\
\hline${ }^{95} \mathrm{Mo}$ & 25 & & 1.808 & 24 & 1.74 & 7.377 & 0 & $\begin{array}{l}1320 \pm 180^{\mathrm{a}} \\
1150 \pm 150^{\mathrm{b}}\end{array}$ & 1270 & 10.60 & -0.45 \\
\hline${ }^{94} \mathrm{Mo}$ & 36 & & 3.204 & 31 & 3.08 & & & & & 10.15 & 0.65 \\
\hline${ }^{93} \mathrm{Mo}$ & 40 & 1 & 2.743 & 73 & 3.10 & 8.092 & 0 & $\begin{array}{l}2700 \pm 500^{\mathrm{a}} \\
2170 \pm 250^{\mathrm{b}}\end{array}$ & 2420 & 9.53 & -0.10 \\
\hline${ }^{92} \mathrm{Mo}$ & 36 & & 4.346 & 36 & 4.35 & & & & & 9.40 & 1.60 \\
\hline${ }^{91} \mathrm{Mo}$ & 23 & 8 & 2.345 & 25 & 2.49 & & & & & 9.00 & -0.16 \\
\hline${ }^{90} \mathrm{Mo}$ & 22 & & 3.185 & 22 & 3.19 & & & & & 9.00 & 0.68 \\
\hline${ }^{100} \mathrm{Nb}$ & 7 & & 0.704 & 7 & 0.70 & & & & & 13.50 & -0.45 \\
\hline${ }^{99} \mathrm{Nb}$ & 12 & 4 & 1.015 & 12 & 1.02 & & & & & 12.50 & -0.45 \\
\hline${ }^{98} \mathrm{Nb}$ & 7 & & 0.737 & 9 & 0.91 & & & & & 13.20 & -0.40 \\
\hline${ }^{97} \mathrm{Nb}$ & 19 & 6 & 2.113 & 19 & 2.11 & & & & & 11.00 & 0.10 \\
\hline${ }^{96} \mathrm{Nb}$ & 9 & & 0.868 & 11 & 1.05 & 6.893 & $9 / 2$ & $46 \pm 5^{b}$ & 45.8 & 13.30 & -0.30 \\
\hline${ }^{95} \mathrm{Nb}$ & 12 & 8 & 1.430 & 19 & 1.71 & 8.489 & 6 & $48 \pm 24^{\mathrm{b}}$ & 48.4 & 11.45 & -0.20 \\
\hline${ }^{94} \mathrm{Nb}$ & 41 & 0 & 1.263 & 31 & 1.09 & 7.232 & $9 / 2$ & $\begin{array}{l}80 \pm 10^{\mathrm{a}} \\
59 \pm 3^{\mathrm{b}}\end{array}$ & 81.9 & 11.00 & -1.20 \\
\hline${ }^{93} \mathrm{Nb}$ & 36 & 5 & 1.813 & 30 & 1.68 & & & & & 9.90 & -0.80 \\
\hline${ }^{92} \mathrm{Nb}$ & 29 & 3 & 1.650 & 41 & 1.88 & & & & & 9.60 & -0.92 \\
\hline${ }^{91} \mathrm{Nb}$ & 29 & 4 & 2.660 & 24 & 2.41 & & & & & 9.60 & 0.00 \\
\hline${ }^{90} \mathrm{Nb}$ & 17 & & 1.195 & 26 & 1.43 & & & & & 8.80 & -1.30 \\
\hline${ }^{99} \mathrm{Zr}$ & 22 & & 1.080 & 22 & 1.08 & & & & & 12.00 & -0.80 \\
\hline${ }^{98} \mathrm{Zr}$ & 11 & & 2.104 & 11 & 2.10 & & & & & 11.65 & 0.50 \\
\hline${ }^{97} \mathrm{Zr}$ & 11 & & 2.265 & 12 & 2.51 & 5.629 & 0 & $\begin{array}{l}13000 \pm 3000^{\mathrm{a}} \\
12100 \pm 2500^{\mathrm{b}}\end{array}$ & 13000 & 11.85 & 0.90 \\
\hline${ }^{96} \mathrm{Zr}$ & 19 & & 3.249 & 19 & 3.25 & 7.856 & $5 / 2$ & $247 \pm 40^{\mathrm{b}}$ & 245 & 12.60 & 1.57 \\
\hline${ }^{95} \mathrm{Zr}$ & 13 & & 2.025 & 15 & 2.12 & 6.507 & 0 & $\begin{array}{l}3200 \pm 800^{\mathrm{a}} \\
3250 \pm 140^{\mathrm{b}}\end{array}$ & 3240 & 11.50 & 0.47 \\
\hline${ }^{94} \mathrm{Zr}$ & 22 & & 3.059 & 19 & 2.95 & 8.220 & $5 / 2$ & $\begin{array}{l}160 \pm 15^{\mathrm{a}} \\
740 \pm 240^{\mathrm{b}}\end{array}$ & 313 & 11.00 & 1.00 \\
\hline${ }^{93} \mathrm{Zr}$ & 21 & & 2.185 & 21 & 2.19 & 6.785 & 0 & $\begin{array}{l}3500 \pm 800^{\mathrm{a}} \\
4880 \pm 580^{\mathrm{b}}\end{array}$ & 4250 & 10.30 & 0.00 \\
\hline${ }^{92} \mathrm{Zr}$ & 42 & & 3.500 & 42 & 3.50 & 8.647 & $5 / 2$ & $\begin{array}{l}550 \pm 100^{\mathrm{a}} \\
618 \pm 54^{\mathrm{b}}\end{array}$ & 607 & 9.50 & 0.67 \\
\hline${ }^{91} \mathrm{Zr}$ & 33 & & 3.007 & 36 & 3.05 & 7.260 & 0 & $\begin{array}{l}6000 \pm 1400^{\mathrm{a}} \\
7670 \pm 990^{\mathrm{b}}\end{array}$ & 6760 & 9.60 & 0.35 \\
\hline${ }^{90} \mathrm{Zr}$ & 37 & & 4.710 & 37 & 4.71 & & & & & 9.00 & 1.80 \\
\hline${ }^{89} \mathrm{Zr}$ & 26 & 4 & 2.493 & 38 & 2.78 & & & & & 9.00 & -0.14 \\
\hline${ }^{88} \mathrm{Zr}$ & 27 & & 3.484 & 22 & 3.30 & & & & & 8.55 & 0.63 \\
\hline
\end{tabular}

a RIPL Obninsk file [44].

${ }^{b}$ RIPL Beijing file [44]. 


\section{Charged-particle OM parameters}

The calculation of the proton transmission coefficients was carried out by taking into account the systematic but anomalous $A$ dependence of the surface imaginary potential depth which was found by Johnson et al. [51] by analyzing low energy $(p, n)$ reactions on 14 nuclides from $A=89$ to 130. The confidence in this behavior has increased for the region $A=900-100$ [52] and is further supported by Grimes [53] through calculations of the two-particle one-hole state densities of these nuclei. Moreover, Lagrange [54] has also taken into account the analysis of proton elastic scattering data at higher energies and found that a strong energy dependence of the imaginary potential depth can reproduce both the low-energy anomaly and the higher-energy global parameters of Perey [55]. Therefore, we have adopted the proton OMP of Johnson et al. [51] with the energy dependence of the imaginary potential depths suggested by Lagrange [54], based on the analysis of the ${ }^{93} \mathrm{Nb}(p, n)^{93}$ Mo reaction cross-section data [51] up to $E_{p}=5.5 \mathrm{MeV}$ and the available total proton reaction cross sections around $E_{p}=10 \mathrm{MeV}$ [55].

Moreover, we found that the use of this local OM parameter set removes the discrepancies between the experimental and $\mathrm{HF}$ calculated excitation functions of the reaction ${ }^{93} \mathrm{Nb}(p, \gamma){ }^{94} \mathrm{Mo}$ above $3.5 \mathrm{MeV}$ that were reported recently by Harissopulos et al. [56]. They found these data, at the astrophysically relevant low proton energies, to be particularly sensitive to both neutron- and proton-nucleus OMPs, whereas we found that the above mentioned discrepancies were related to uncertainties in the determination of nuclear level densities in this energy region. For the relevant nuclei, there are no data for the $s$-wave neutron resonance spacings at the neutron separation energy.

Our HF results obtained by using the NLD adopted in the present work, and their comparison with the experimental data for both the ${ }^{93} \mathrm{Nb}(p, \gamma)^{94}$ Mo reaction at $E_{p}=1.4-4.9 \mathrm{MeV}$ [56] and the ${ }^{93} \mathrm{Nb}(p, n)^{93}$ Mo reaction up to $E_{p}=5.5 \mathrm{MeV}$ [51] over more than six orders of magnitude are shown in Fig. 1. The absolute values as well as the behavior of these excitation functions are well described within the limits of the error bars and the width of the energy bins involved in the average description of the nuclear excited continuum, i.e., 0.15-0.2 MeV. A bump appears in the measured $(p, \gamma)$ excitation function around $E_{p}=3.5 \mathrm{MeV}$. This behavior is well reproduced by the presently employed local OMPs that were tuned to the lower total neutron cross sections for Mo isotopes below $1 \mathrm{MeV}$ [48], whereas it is not reproduced by the global OMs of Wilmore and Hodgson [57], Perey [55], or Becchetti and Greenlees [58].

The optical model potential for calculation of $\alpha$-particle transmission coefficients was established previously by analysis of the experimental $(n, \alpha)$ reaction cross sections just above the effective Coulomb barrier [60]. Finally, the charged-particle transmission coefficients involved in this work have been checked by looking for a good description of the $(n, p)$ and $(n, \alpha)$ reaction cross sections, respectively, in the first 3-5 MeV above threshold, where their effect is largest. Since the nuclear level density of the corresponding residual nucleus plays no role in this energy range, and the

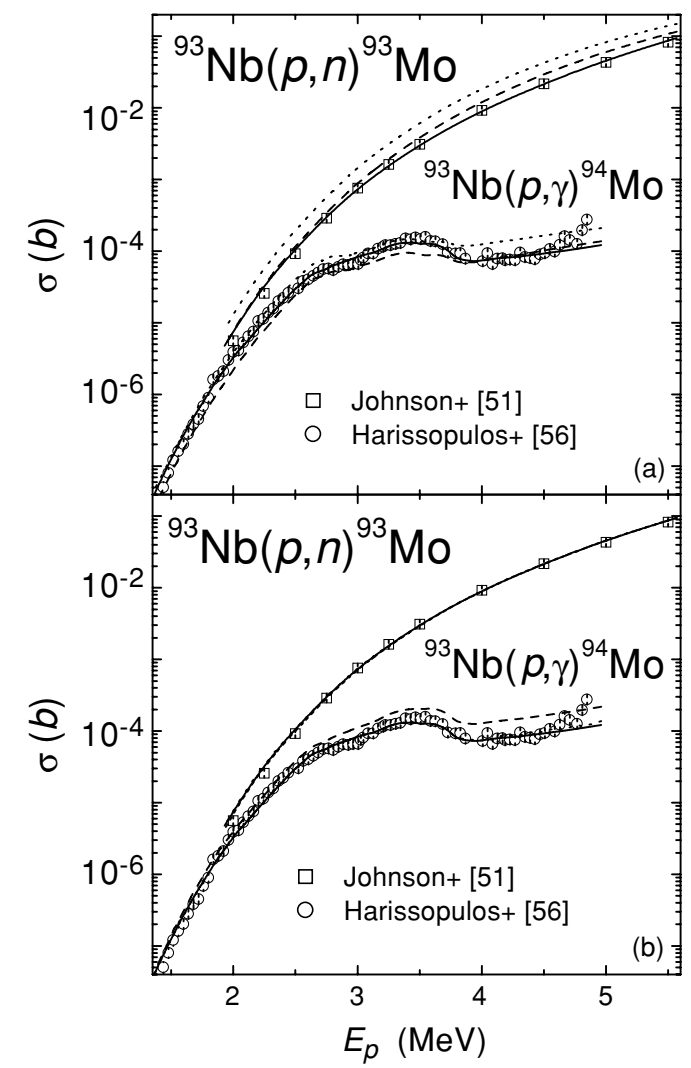

FIG. 1. Comparison of the calculated cross sections for the reaction ${ }^{93} \mathrm{Nb}(p, n){ }^{93} \mathrm{Mo}$ up to $E_{p}=5.5 \mathrm{MeV}$ [51], and the reaction ${ }^{93} \mathrm{Nb}(p, \gamma){ }^{94} \mathrm{Mo}$ at $E_{p}=1.4-4.9 \mathrm{MeV}$ [56] by using the OMPs and NLD described in the text (solid curves). Part (a) furthermore shows comparisons with calculations using the global OMPs of Wilmore and Hodgson [57] and Perey [55] for neutrons and protons, respectively (dashed curves), and Becchetti and Greenlees [58] (dotted curves). Part (b) shows the results of calculations with the NLD $a$ parameter of the nucleus ${ }^{94} \mathrm{Mo}$ increased by $10 \%$ in the limit of the prediction accuracy of the smooth curve method [59], corresponding to a twice lower $s$-wave neutron resonance spacing (dashed curves), and the NLD of the nucleus ${ }^{93}$ Mo obtained by a similar fit of the resonance data [44] but a virtual ground-state shift reduced by $100 \mathrm{keV}$ which may correspond to an alternative fit of the low-lying levels (dotted curves-nearly coincident with solid curves). The experimental data are taken from the EXFOR data file (e.g., see Ref. [46]).

PE contribution is still negligible, a good description of the experimental data in this case may be considered as a validation of the adopted OMPs (see Section IV).

The calculation of the deuteron transmission coefficients has been carried out by using the global OMPs of Lohr and Haeberli [61] and validated through the analysis of the deuteron-emission spectra at 14.8 MeV [62].

\section{The $\gamma$-ray strength functions}

The $\gamma$-ray transmission coefficients were obtained by using for the dominant electric dipole transition strength function $f_{E 1}\left(\varepsilon_{\gamma}\right)$ the giant dipole resonance (GDR) model with an energy-dependent Breit-Wigner line shape $[63,64]$. 


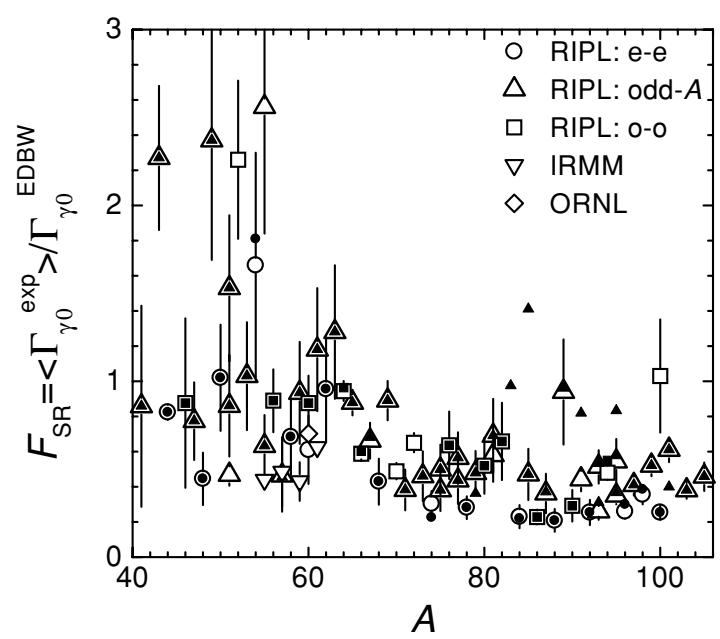

FIG. 2. The EDBW model correction factors provided by the ratio of experimental average radiative width of the $s$-wave neutron resonances, $\Gamma_{\gamma 0}^{\exp }$, to the EDBW predicted values, for nuclei in the mass range $40<A<105$. The experimental data are from RIPL [44] (open and solid symbols correspond to the Obninsk and Beijing files, respectively) and recent measurements at IRMM and ORNL [65].

The usual normalization procedure of $f_{M 1}, f_{E 2}, \ldots$, relative to the dominant $E 1$ contribution was applied in order to calculate the radiative width $\Gamma_{\gamma 0}^{\mathrm{EDBW}}$ of the $s$-wave neutron

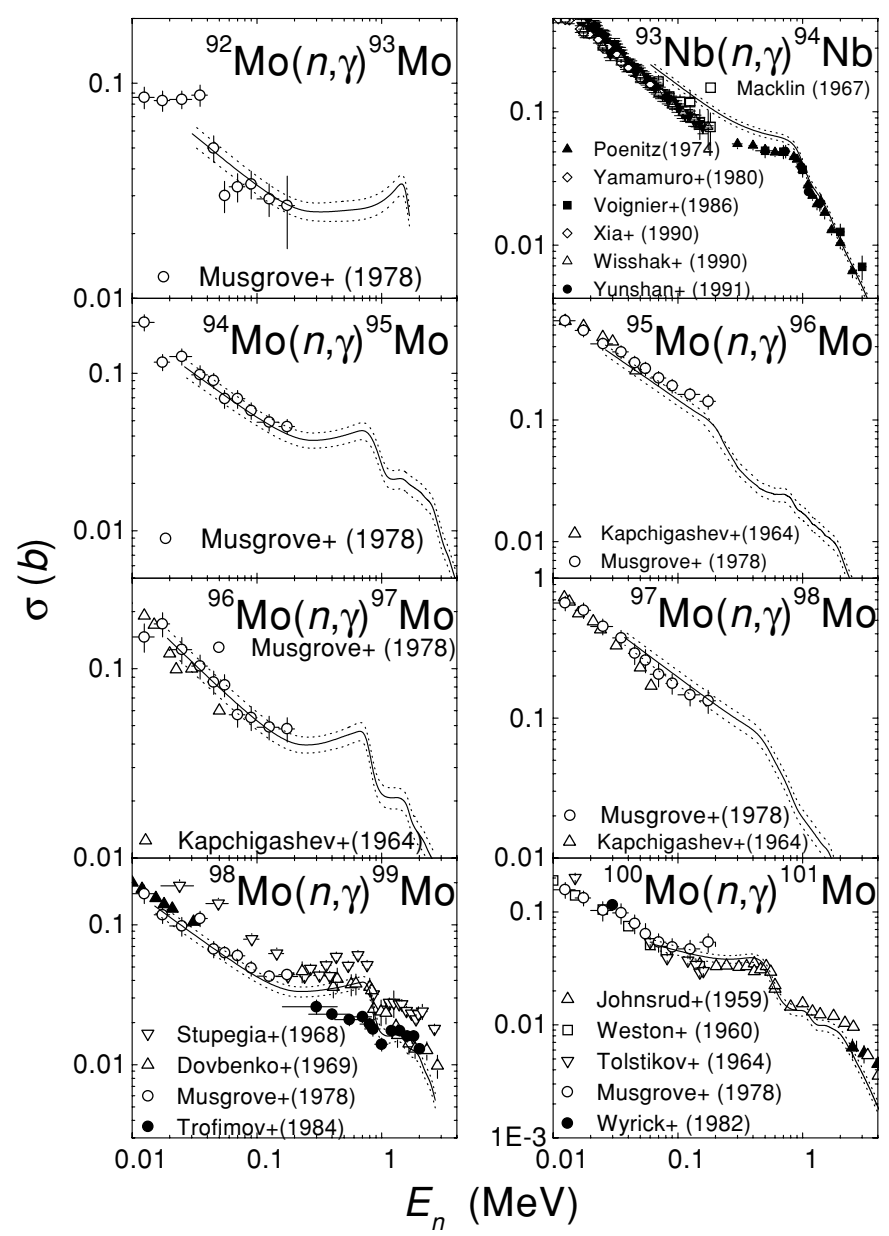

resonances. Systematic EDBW model correction factors were established in the range $A=41-105$ assuming that they are given by the ratio $F_{\mathrm{SR}}=\Gamma_{\gamma 0}^{\mathrm{exp}} / \Gamma_{\gamma 0}^{\mathrm{EDBW}}$ (see Fig. 2). The experimental average values $\Gamma_{\gamma 0}^{\exp }$ were obtained from Refs. $[44,65]$.

This method was validated by comparison of calculated and experimental capture cross sections of ${ }^{59} \mathrm{Co}$ and ${ }^{58,60} \mathrm{Ni}$ [66], and ${ }^{93} \mathrm{Nb}$ and ${ }^{92,94-98,100} \mathrm{Mo}$ (see Fig. 3) in the neutron energy range from a few $\mathrm{keV}$ to $2-3 \mathrm{MeV}$. The corresponding $\mathrm{HF}$ calculations have obviously been performed by means of the same neutron OMPs and NLD parameters as for the rest of the activation cross-section calculations. The use of RIPL data for $A \sim 90$ has provided a suitable HF description of the capture data. This contrasts with the case of the mass region $A \sim 60$ where the RIPL values for $\Gamma_{\gamma 0}^{\text {exp }}$ lead to $\gamma$-ray strength functions that are too large, whereas recent values [65] provide good agreement with the experimental capture data [31]. Thus, the correctness of the $\gamma$-ray strength functions was ensured, which is quite important for a correct account of the isomeric-state cross sections.

\section{Preequilibrium treatment}

The preequilibrium (PE) cross sections have been calculated with a generalized version of the geometry-dependent hybrid (GDH) model [67], which includes up-to-date
FIG. 3. Comparison of experimental and calculated capture cross sections of ${ }^{93} \mathrm{Nb}$ and ${ }^{92,94-98,100} \mathrm{Mo}$ for neutron energies up to 2-3 MeV. The solid curves have been obtained with the EDBW model correction factors corresponding to the experimental average radiative width of the $s$-wave neutron resonances $\Gamma_{\gamma 0}^{\exp }[44,65]$, while the dotted curves correspond to the error bar of $\Gamma_{\gamma 0}^{\exp }$. The experimental data are taken from the EXFOR data file [46]. 


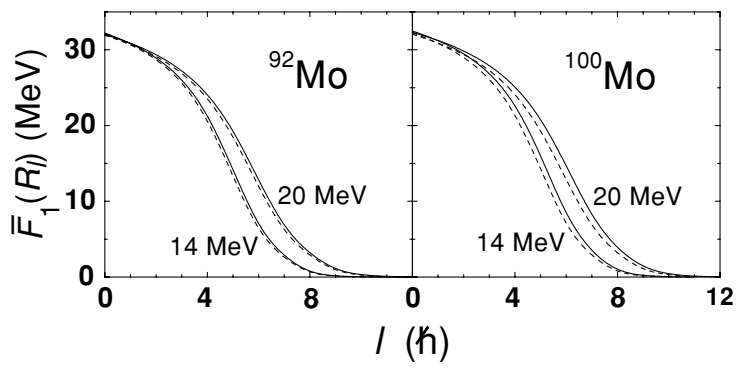

FIG. 4. Local-density Fermi energies for neutrons (solid curves) and protons (dashed curves) versus incident partial wave for incident neutrons on the target nuclei ${ }^{92,100} \mathrm{Mo}$ at 14 (lower curves) and $20 \mathrm{MeV}$ (upper curves). The central well Fermi energy is $F_{0}=40 \mathrm{MeV}$.

particle-hole level densities and angular momentum conservation [33], as well as $\alpha$-particle and deuteron emission.

Particle-hole (p-h) level densities $\rho(p, h, E)$ were determined by a revised version of the subroutine PLD [68] using the Fermi-gas model (FGM) energy dependence of the single-particle level (s.p.l.) density [69]. The Fermi energies $\bar{F}_{1}\left(R_{l}\right)$ (see Fig. $4 ; R_{l}=l \bar{\lambda}$ ) that determine the finite-depth correction to $\rho(p, h, E)$ for the first nucleon-nucleon interaction $(h=1)$ were determined as a function of orbital angular momentum by a trajectory average in the local density approximation using an average imaginary optical model potential ( $[6,67,70]$ and Eqs. 58 and 59 of Ref. [68]). In such an approach, the first interaction predominantly takes place near the surface (surface effect [71]).

The energy dependence of the PE contribution in this approach is affected by the successive openings of contributions from different partial waves. In this model, the onset of partial wave contributions is sharp, since additional p-h states are included as soon as a particular $\bar{F}_{1}\left(R_{l}\right)$ crosses the hole excitation energy. Here, such sharp, unphysical increases are eliminated by a smoothing of the contribution over about $2 \mathrm{MeV}$. Neutron and proton $l=4 \hbar$ partial wave contributions of ${ }^{92,94,95,97}$ Mo start around 8.7, 8.1, 8.3, and $8.5 \mathrm{MeV}$, respectively, whereas for the $l=5 \hbar$ partial wave, the start is around 15.7, 14.9, 15.2, 14.1, 14.8, and $13.3 \mathrm{MeV}$ for ${ }^{92,94-98}$ Mo. Because of the energy dependence of the PE strength, only the latter significantly modifies the calculated excitation functions, e.g., for $(n, p)$ reactions (see below).

For preequilibrium $\alpha$-particle emission we adopted the relation $g_{\alpha}=A / 10.36 \mathrm{MeV}^{-1}$ [72] for the $\alpha$-particle state density. As in Ref. [6], we adopted $\bar{F}_{1}^{\alpha}\left(R_{l}\right)=4 \bar{F}_{1}\left(R_{l}\right)$, leading to onsets of $l=6 \hbar$ partial wave contributions for ${ }^{92,98,100} \mathrm{Mo}$ around $12.5 \mathrm{MeV}$ and of $l=7 \hbar$ around 19.5, 18.3, and $18.4 \mathrm{MeV}$, respectively. A free parameter in this approach is the $\alpha$-particle preformation probability. This parameter was fixed at 0.18 , which is the value required to reproduce the measured $\alpha$-particle emission spectra induced by $14.8 \mathrm{MeV}$ neutrons [62].

For preequilibrium deuteron emission, we have adopted, as a first trial, a simple extrapolation of the Milano group method for the case of the $\alpha$-particle PE emission (see e.g.,
[73]). The corresponding s.p.l. density at the saturated Fermi energy is assumed as twice that of the $g_{\alpha}$ value and $\bar{F}_{1}^{d}\left(R_{l}\right)=$ $2 \bar{F}_{1}\left(R_{l}\right)$. The deuteron preformation probability is a free parameter.

\section{RESULTS AND DISCUSSION}

The experimental results of this work are presented in Tables II and III. They are shown graphically in Figs. 5-9 in order of ascending mass of the target nucleus. Channels leading to the same activity in the case of the IRMM measurements were separated by use of a combination of natural and enriched samples, as described in Ref. [5]. Low-energy corrections for the highest incident deuteron energies are partly based on the present model calculations (local approach) for the required excitation function below $10 \mathrm{MeV}$. Thus, above $16 \mathrm{MeV}$, unique new results were obtained for the ${ }^{92} \mathrm{Mo}(n, 2 n)^{91} \mathrm{Mo}^{\mathrm{m}},{ }^{92} \mathrm{Mo}(n, \alpha)^{89} \mathrm{Zr}^{\mathrm{m}},{ }^{94} \mathrm{Mo}(n, 2 n)^{93} \mathrm{Mo}^{\mathrm{m}}$, ${ }^{95} \mathrm{Mo}(n, p){ }^{95} \mathrm{Nb}^{\mathrm{m}},{ }^{96} \mathrm{Mo}(n, x){ }^{95} \mathrm{Nb}^{\mathrm{m}},{ }^{97} \mathrm{Mo}(n, p){ }^{97} \mathrm{Nb},{ }^{97} \mathrm{Mo}(n$, p) $\left.{ }^{97} \mathrm{Nb}^{\mathrm{m}},{ }^{97} \mathrm{Mo}(n, x){ }^{96} \mathrm{Nb},{ }^{98} \mathrm{Mo}(n, p)\right)^{98} \mathrm{Nb}^{\mathrm{m}},{ }^{98} \mathrm{Mo}(n, x){ }^{97} \mathrm{Nb}$, ${ }^{98} \operatorname{Mo}(n, x){ }^{97} \mathrm{Nb}^{\mathrm{m}}$, and ${ }^{100} \mathrm{Mo}(n, 2 n){ }^{99}$ Mo reactions, whereas good accuracy results were added to the few previous results for the ${ }^{92} \mathrm{Mo}(n, p){ }^{92} \mathrm{Nb}^{\mathrm{m}}$, ${ }^{96} \mathrm{Mo}(n, p){ }^{96} \mathrm{Nb}$, and ${ }^{100} \mathrm{Mo}(n$, $\alpha)^{97} \mathrm{Zr}$ reactions.

A significant body of new good-accuracy $14 \mathrm{MeV}$ data has resulted from the use of natural samples at KRI. For a considerable number of the reactions studied, these data can be compared with the results obtained at IRMM at higher energies. For the ${ }^{92} \mathrm{Mo}(n, 2 n){ }^{91} \mathrm{Mo}^{\mathrm{m}},{ }^{92} \mathrm{Mo}(n, p){ }^{92} \mathrm{Nb}^{\mathrm{m}},{ }^{92} \mathrm{Mo}(n, \alpha){ }^{89} \mathrm{Zr}^{\mathrm{m}}$, ${ }^{94} \mathrm{Mo}(n, 2 n){ }^{93} \mathrm{Mo}^{\mathrm{m}}, \quad{ }^{98} \mathrm{Mo}(n, p){ }^{98} \mathrm{Nb}^{\mathrm{m}}, \quad{ }^{100} \mathrm{Mo}(n, 2 n){ }^{99} \mathrm{Mo}$, and ${ }^{100} \operatorname{Mo}(n, \alpha){ }^{97} \mathrm{Zr}$ reactions, the data obtained with natural samples require no correction for sample composition, and the KRI results are in excellent agreement with the extrapolated trend of the results obtained at IRMM. In the case of the ${ }^{95} \mathrm{Mo}(n, p){ }^{95} \mathrm{Nb}^{\mathrm{m}},{ }^{96} \mathrm{Mo}(n, p){ }^{96} \mathrm{Nb}$, and ${ }^{97} \mathrm{Mo}(n, p){ }^{97} \mathrm{Nb}$ reactions, corrections based on the present local approach result in a similar excellent agreement for the latter two reactions and improved agreement for the first. Corrections based on the global approach lead to similar conclusions, except in the case of the ${ }^{97} \mathrm{Mo}(n, p){ }^{97} \mathrm{Nb}$ reaction, where the corrected results appear to be too low. The KRI data shown in Figs. 5-9 have been corrected for the reaction on a neighboring nuclide using reaction cross sections obtained with the present global approach, whenever this was appropriate (see Table III).

It is clear that now a firm experimental database exists above $13 \mathrm{MeV}$ for the cross sections of the dominant neutron, proton, and $\alpha$ emission channels. Around $14.5 \mathrm{MeV}$, this database is complemented by emission spectra [62]. The experimental data below $13 \mathrm{MeV}$ have been measured mostly at FZ Jülich, and to some extent at Debrecen, but in general, the low-energy region has not been investigated as well as the higher-energy region. Nonetheless, the database now available suggests a careful model analysis which is described in detail below. A detailed discussion of the comparison of model calculations with the measured data is given in the sections below. Here we note that with regard to the model calculations in the global approach, we see a good overall agreement with the 


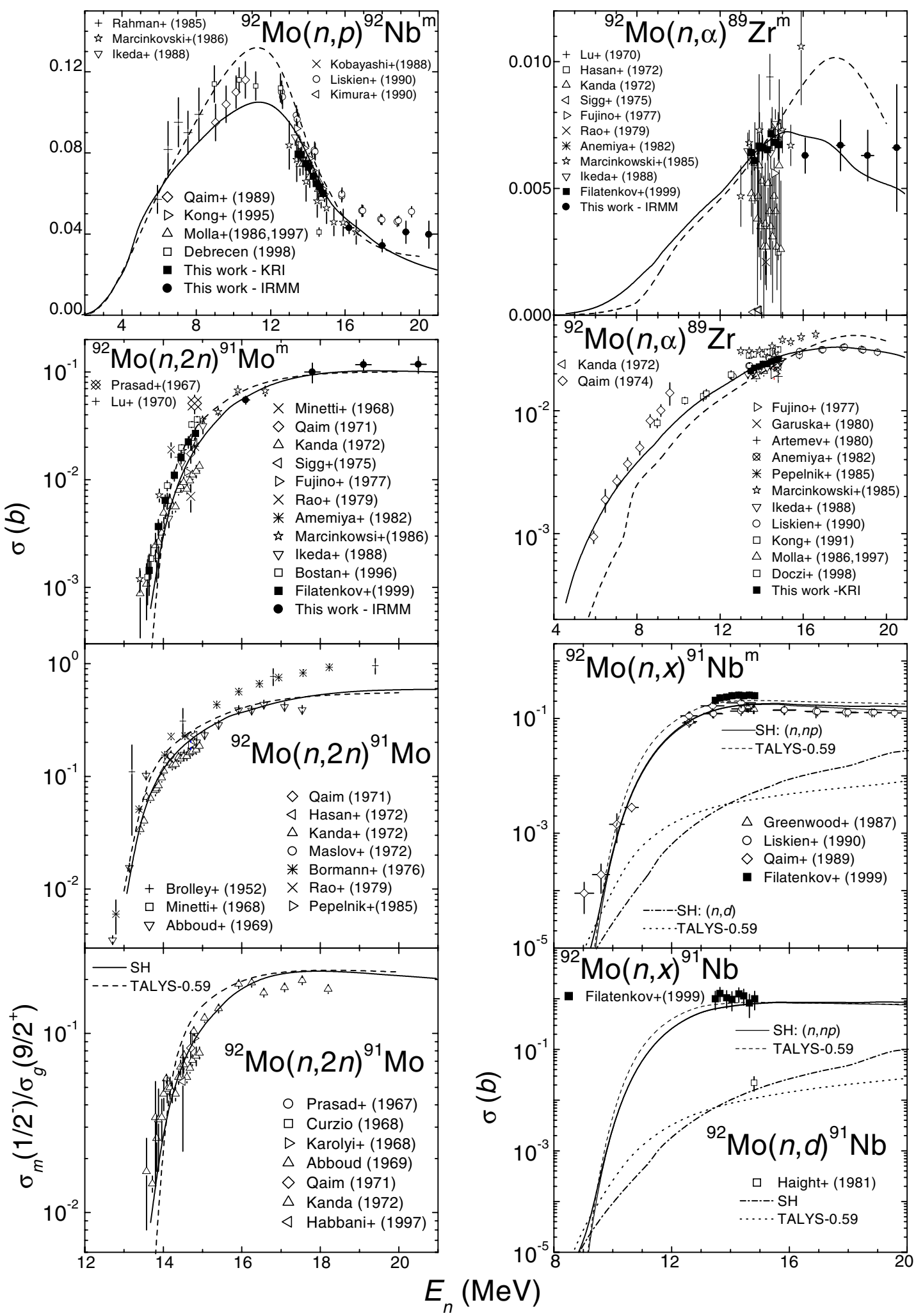

FIG. 5. Comparison of experimental cross sections for the indicated channel with calculations using the STAPRE-H (solid and dot-dash curves) and TALYS (dashed and dotted curves) codes for the target nucleus ${ }^{92} \mathrm{Mo}$. Unless explicitly indicated by a superscript $m$ for isomer or a superscript $g$ for ground-state cross sections, the sum of the two residual nuclide contributions is given. The experimental data are taken from the EXFOR data file [46]. 

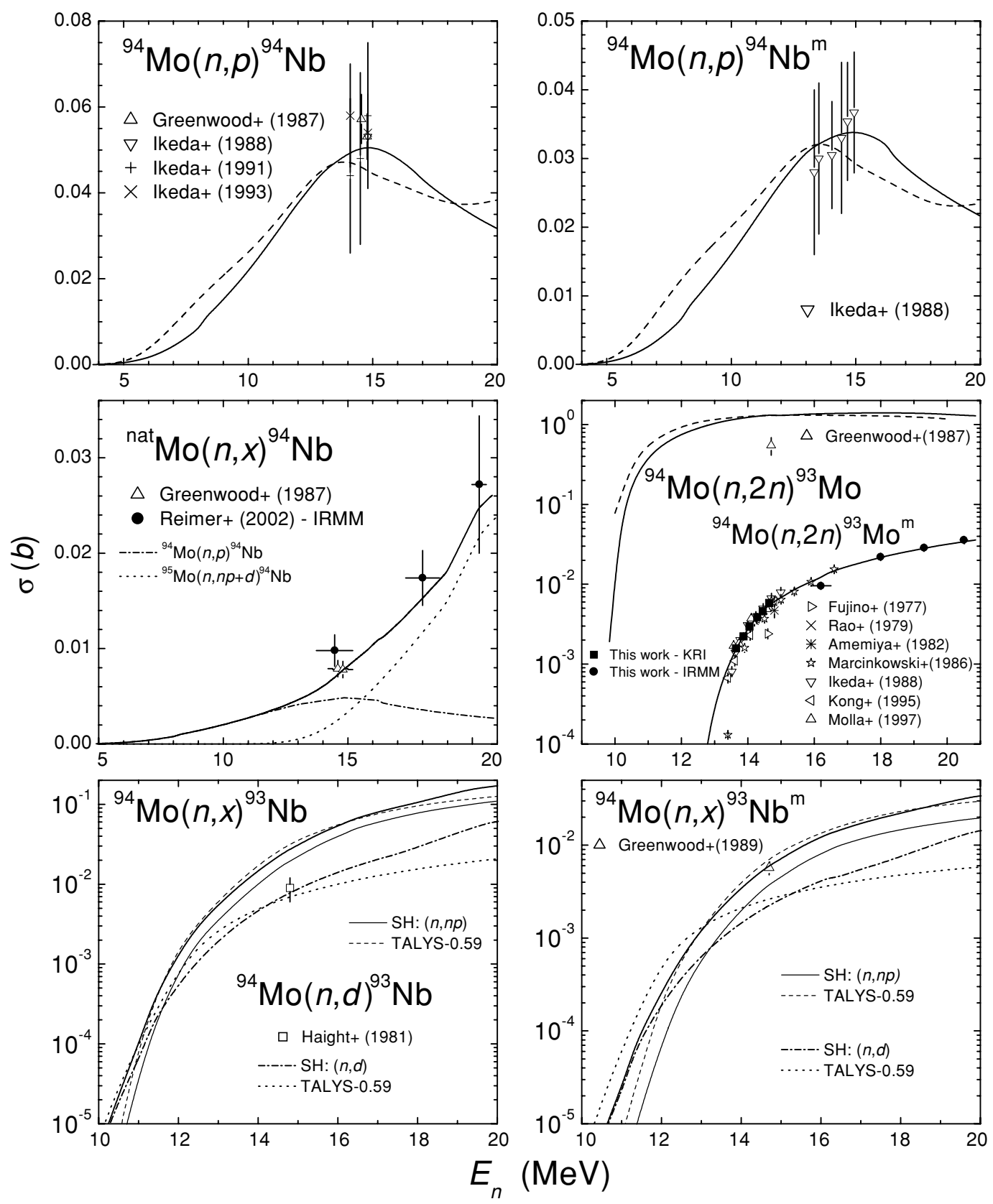

FIG. 6. As for Fig. 5, but for the target nucleus ${ }^{94}$ Mo.

data for the total activation cross sections, except in the case of $(n, \alpha)$ reactions for the heavy Mo isotopes. For reactions leading to isomers, the deviations are not always negligible.

With regard to the model calculations in the local approach, we make the following general observations.

(i) The rise in the excitation functions is satisfactorily reproduced in all cases except for local disagreements at some energies that are probably due to limited knowledge of the corresponding low-lying levels. This supports the validity of the transmission coefficients used for the respective particles. (ii) The agreement between experimental and calculated data is, in general, satisfactory both at the maxima and at the tails of the excitation functions. This essentially means that the contributions due to both PE emission and equilibrated system decay are reasonably reproduced.

\section{A. The $(n, p)$ reactions}

The new measured data concern the total $(n, p)$ cross sections (ground state plus isomer) for ${ }^{95,96,97} \mathrm{Mo}$ at KRI and ${ }^{96,97} \mathrm{Mo}$ at IRMM, as well as the isomer cross sections for ${ }^{92,95,97,98} \mathrm{Mo}$ at IRMM and ${ }^{92,95,98} \mathrm{Mo}$ at KRI. For the sake of comparison with the model calculations, the available data for ${ }^{94}$ Mo total and isomer cross sections are shown as well. 

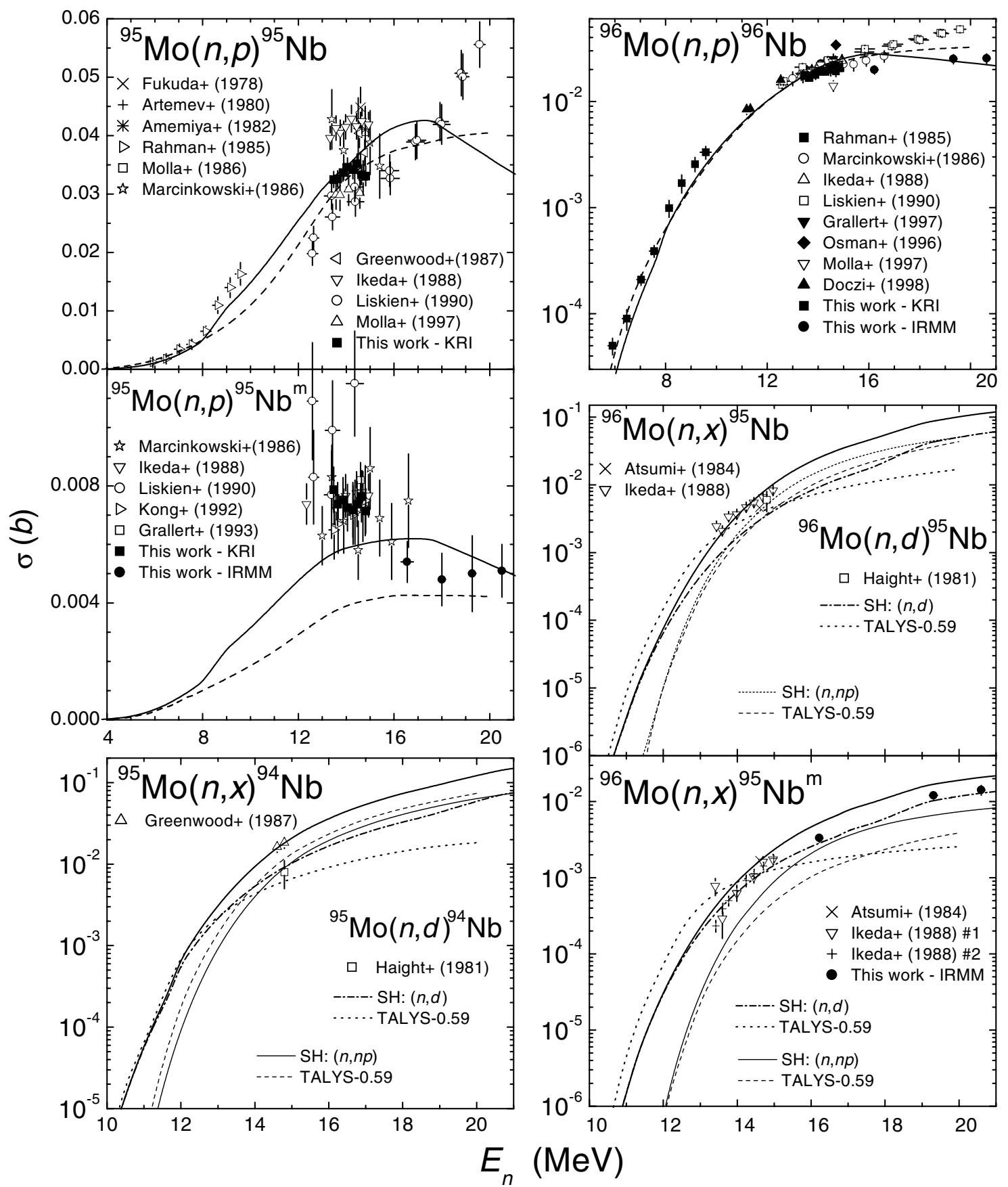

FIG. 7. As for Fig. 5, but for the target nuclei ${ }^{95,96} \mathrm{Mo}$.

\section{Total activation cross sections}

For ${ }^{95} \mathrm{Mo}$, the new KRI measurements around $14 \mathrm{MeV}$ are seen to be badly needed in order to shed light on the discrepancies of earlier measurements around this energy, which group around two values. Both the global and the local approach agree very well with the new KRI results. Above $14 \mathrm{MeV}$, the two approaches give similar results, even if the shapes of the excitation curves are seen to be somewhat different. The large differences from the measurements by Liskien et al. [18] are a consequence of the use of natural samples by the latter, which means their measured cross sections include contributions from the ${ }^{96} \mathrm{Mo}(n, x){ }^{95} \mathrm{Nb}$ reaction. This indicates that additional measurements would be required to unambiguously establish the excitation curve. Below $10 \mathrm{MeV}$, the local approach agrees best with the data of Rahman and Qaim [74].

In the case of ${ }^{96} \mathrm{Mo}$, the new KRI measurements essentially confirm earlier measurements around $14 \mathrm{MeV}$. The new IRMM measurements agree with those $14 \mathrm{MeV}$ data while showing a much lower cross section near $20 \mathrm{MeV}$ than was given earlier by Liskien et al. [18]. Again, this may be attributed 

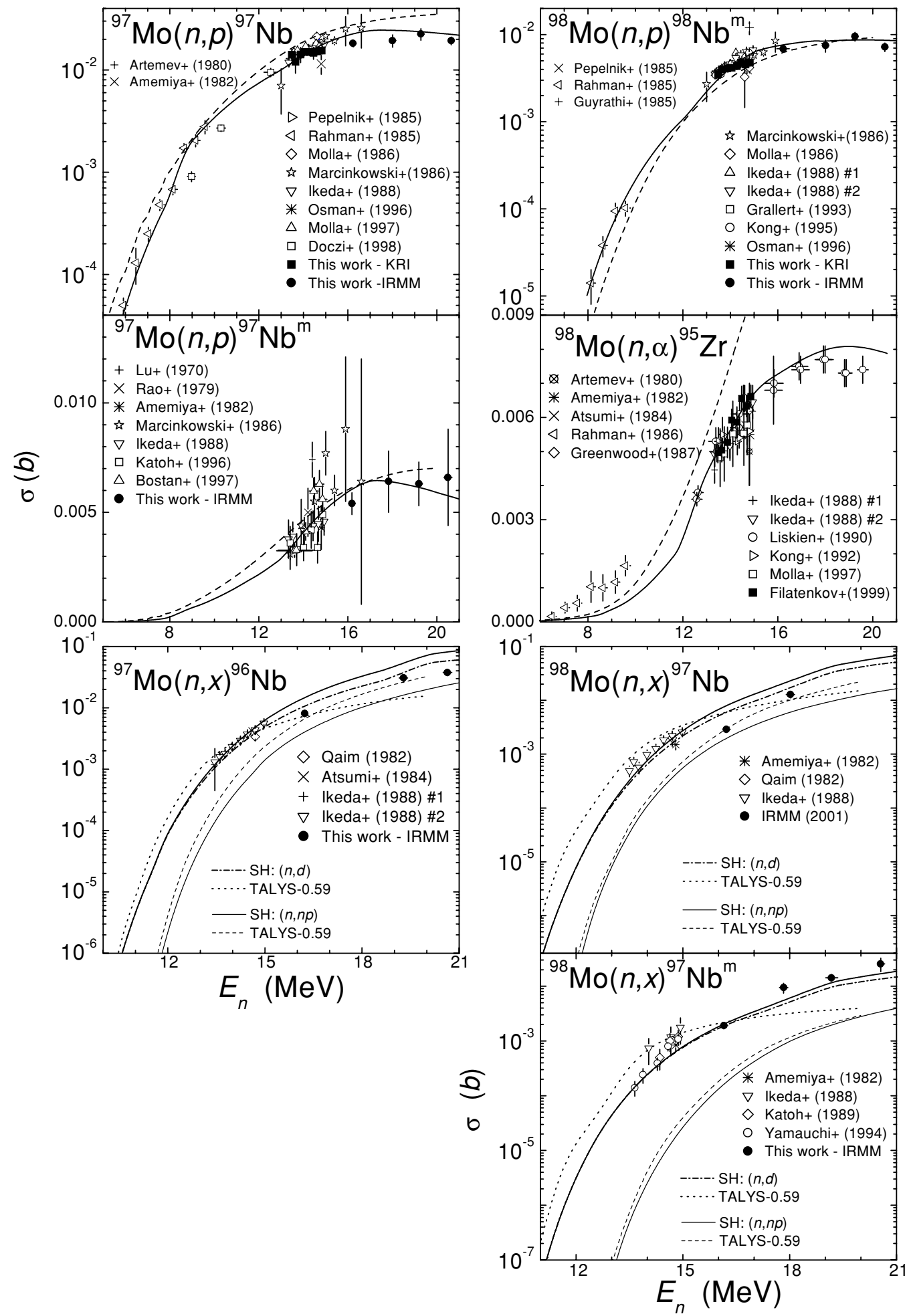

FIG. 8. As for Fig. 5, but for the target nuclei ${ }^{97,98}$ Mo.

to the use of natural samples by the latter. The local and the global approach agree with the measurements by Rahman and Qaim [74] below $10 \mathrm{MeV}$ and with the new IRMM data above $15 \mathrm{MeV}$.
For ${ }^{97} \mathrm{Mo}$, all measurements are in agreement with each other and with the local approach. Agreement with the global approach is good up to $16 \mathrm{MeV}$, above which, however, it is too high by up to $50 \%$. 

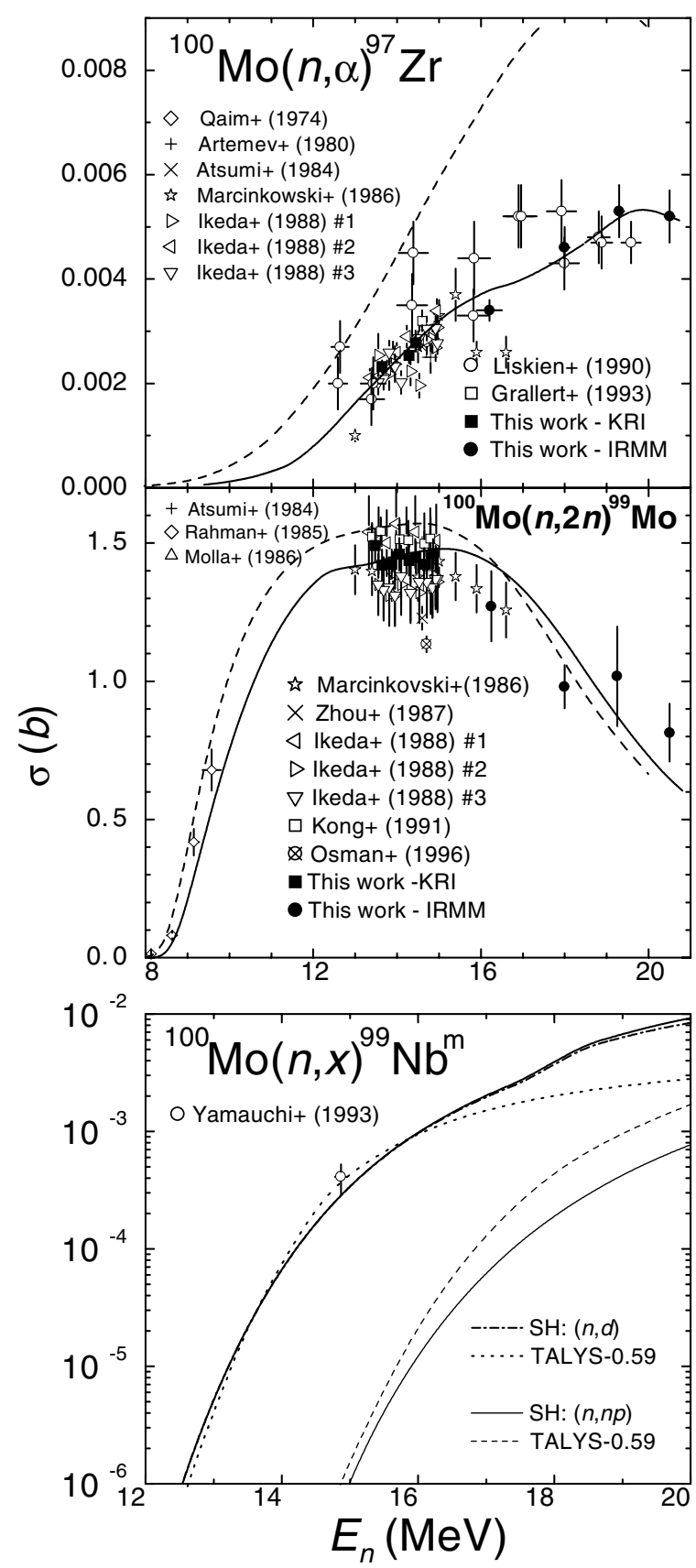

FIG. 9. As for Fig. 5, but for the target nucleus ${ }^{100}$ Mo.

The case of ${ }^{94} \mathrm{Mo}$, added for comparison, shows agreement of the local approach with the available $14 \mathrm{MeV}$ data, but it also shows a discrepancy in shape with the global approach. Additional measured data below 13 and above $15 \mathrm{MeV}$ are required to study this issue in more detail.

\section{Isomer cross sections}

For ${ }^{92} \mathrm{Mo}$, the KRI measurements agree with the bulk of the available data at $14 \mathrm{MeV}$, and the new IRMM results agree well with the cross sections of Marcinkowski et al. [76] and the shape of the excitation curve suggested by those data. Thus, it appears that the data by Liskien et al. [18] are too high by about $20 \%$, except for the data points at the highest two energies. However, it is worthwhile to point out that for the new data at 19.3 and $20.5 \mathrm{MeV}$, the low-energy correction appears to be underestimated, when the overall shape of the excitation curves suggested by the lower energy data points and the model calculations are considered. The local and global approaches agree well with each other below 9 and above $14 \mathrm{MeV}$ but show a large difference of maximum $25 \%$ between 8 and $14 \mathrm{MeV}$. In the latter region, the local approach is about one standard deviation below the measured data by Qaim et al. [1] and Liskien et al. [18], whereas the global approach is just above those measured data. Above $14 \mathrm{MeV}$, the global approach and the local approach follow the new IRMM and KRI results.

In the case of ${ }^{95} \mathrm{Mo}$, the IRMM data above $16 \mathrm{MeV}$ are unique, and the KRI data around $14 \mathrm{MeV}$ are valuable in view of the large scatter in the measurements performed previously. The local approach shows reasonable agreement with the new data, although it falls about $20 \%$ short of the KRI data. The global approach is about $30 \%$ too low, indicating a problem with the level scheme, $\gamma$-ray strengths, or the level density modeling of the residual nucleus. It may be noted that correction of the KRI data for sample composition with the TALYS calculation would result in values that fall well below the trend suggested by the IRMM data above $16 \mathrm{MeV}$.

For ${ }^{97,98} \mathrm{Mo}$, the IRMM data above $16 \mathrm{MeV}$ are unique, and the trend of the excitation curve is in good agreement with the measurements at lower energy. The KRI data around $14 \mathrm{MeV}$ for ${ }^{98}$ Mo show that the cross section at that energy is well established. The agreement between the local and global approaches and all data is excellent, although unfortunately no measured data are available for ${ }^{97}$ Mo below $13 \mathrm{MeV}$.

Again, for comparison we refer to the data of ${ }^{94} \mathrm{Mo}$. Both the local and the global approach agree with the available data around $14 \mathrm{MeV}$ within the errors. The fairly substantial differences in magnitude and shape of the predicted cross sections suggest the need for further measurements above 15 and below $13 \mathrm{MeV}$.

\section{B. The $(n, \alpha)$ reactions}

New data for total activation $\left({ }^{92,100} \mathrm{Mo}\right.$, ground state plus isomer) and for isomer cross sections $\left({ }^{92} \mathrm{Mo}\right)$ were obtained.

The new KRI data for the total $(n, \alpha)$ cross section of ${ }^{92}$ Mo favor earlier measurements by Doczi et al. [77], Molla et al. [78], and Liskien et al. [18], whereas they are distinctly below the values proposed by Marcinkowski et al. [76] and Kong et al. [79]. The newly measured data and the available data generally agree well with the local approach, except around $9 \mathrm{MeV}$ where the data of Doczi et al. [77] and Rahman et al. are well above the calculated values. The global approach is at odds with the measured excitation curve. The problem lies, most likely, with the transmission coefficients for the $\alpha$ particle, but also with the preequilibrium model. For complex particle emission, the phenomenological stripping, pickup, and knockout model of Kalbach [75] has been adopted, and this model is mostly tuned to emission spectra for nucleon-induced reactions above $20 \mathrm{MeV}$. 
Indeed, a global comparison of all $(n, \alpha)$ data across the periodic table reveals some overestimation for heavy nuclides. Clearly, the model needs to be readjusted to give a better agreement at lower incident energies. Again, as with the local approach in this paper, a local adjustment of the preequilibrium parameters (in this case the spectroscopic strength) would remedy the deviation.

For the total $(n, \alpha)$ cross section of ${ }^{100} \mathrm{Mo}$, the new data at $14 \mathrm{MeV}$ (KRI) and above (IRMM) suggest a much better defined measured excitation curve than was previously the case. The new data are well reproduced by the local approach. On the other hand, the deviations with the global approach are considerable, emphasizing the need for further model adjustments similar to those mentioned above for ${ }^{92} \mathrm{Mo}$.

For comparison, the total $(n, \alpha)$ cross section of ${ }^{98} \mathrm{Mo}$ is shown as well. Good agreement is obtained in the local approach above $12 \mathrm{MeV}$, whereas the global approach overestimates the data above $12 \mathrm{MeV}$. Again, differences are large, emphasizing further the arguments presented above.

The new data for the isomer cross section of ${ }^{92} \mathrm{Mo}$ are unique above $14 \mathrm{MeV}$ and serve to shed light on the large spread of the earlier data around $14 \mathrm{MeV}$. The new data agree well with each other and with calculations in the local approach. The global approach agrees with the data at $14 \mathrm{MeV}$ but overshoots the higher-energy data by about $50 \%$.

All measured excitation curves are remarkably "flat" or tend to be even increasing at energies above $15 \mathrm{MeV}$, resulting in shapes that are quite different from the characteristic bell shape, as given, for instance, by the TALYS calculation for the ${ }^{92} \mathrm{Mo}(n, \alpha)^{89} \mathrm{Nb}^{\mathrm{m}}$ reaction. This is particularly evident for 98,100 Mo which exhibit larger PE contributions than ${ }^{92} \mathrm{Mo}$, in agreement with the isotope effect (see, e.g., [84,85]). A comparison between the two approaches shows that the local approach is much better tuned to describe the excitation curves than the global approach. The requirements to improve the latter were presented above. The wiggly features in the STAPRE-H (local approach) calculations for the isomer cross sections of ${ }^{92}$ Mo and for ${ }^{100}$ Mo result from the onsets of partial wave contributions (see Sec. III).

The larger cross section values at the maxima with respect to the previous analysis [13], in which $F_{0}=37 \mathrm{MeV}$ was used, are due to the higher value used here $\left(F_{0}=40 \mathrm{MeV}\right)$. This change results in the lowering of the corresponding $\mathrm{PE}$ "thresholds" and therefore a stronger $\alpha$ particle PE contribution.

\section{The $(n, 2 n)$ reactions}

The total $(n, 2 n)$ cross section measurements for ${ }^{100} \mathrm{Mo}$ are new above $16 \mathrm{MeV}$, agree well with the earlier measurements by Marcinkowski et al. [76] below $16 \mathrm{MeV}$, and match well with the new data by KRI around $14 \mathrm{MeV}$. Both the local and the global approach show good agreement with the measured data, with a slight preference for the former.

The measured ${ }^{92} \operatorname{Mo}(n, 2 n)$ cross sections for the production of the isomer also result in the first data above $16 \mathrm{MeV}$. The excitation curve is experimentally well established from threshold to $20 \mathrm{MeV}$, and the local and global approaches agree well with the measurements in the entire range. For reference, the existing total $(n, 2 n)$ measurements and the ratio of isomer to ground-state production cross sections are shown as well. For the total $(n, 2 n)$ cross sections, measurements are discrepant above $14 \mathrm{MeV}$, and both calculations clearly favor the lower values of Abboud et al. [80]. The measured data for the ratio essentially confirm the estimates by the two approaches. In all, this indicates that the measurements of Bormann et al. [81] and Brolley et al. [82] for the total $(n, 2 n)$ cross sections are too high.

For the isomer produced by the ${ }^{94} \mathrm{Mo}(n, 2 n)^{93} \mathrm{Nb}^{\mathrm{m}}$ reaction, the excitation curve is well established by unique new data above $16 \mathrm{MeV}$, as well as the new and the existing data around $14 \mathrm{MeV}$. The data are well described in the local approach.

A special point for this excitation function concerns the population of the isomeric state, which is the 16th excited level of ${ }^{93} \mathrm{Mo}$ at the excitation energy of $2.425 \mathrm{MeV}$. The rather well known experimental decay scheme of 40 levels up to $2.743 \mathrm{MeV}$ taken into account in the STAPRE-H calculation includes no transition to the isomeric state, so that the calculated results are based fully on the adopted $\gamma$-ray strength functions. The good agreement between the calculated excitation function and the present experimental data within the whole energy range, including the threshold region, proves that the description of the $\gamma$ deexcitation involved within this work is correct.

It may be noted that the calculated total ${ }^{94} \operatorname{Mo}(n, 2 n)$ reaction cross sections agree well with each other but are at variance with the two measurements by Greenwood [83].

\section{The $\Delta Z=1, \Delta A=1$ reactions}

The results of the new measurements include two reactions for the target nuclei ${ }^{96} \mathrm{Mo}$ and ${ }^{98} \mathrm{Mo}$ leading to an isomer and two total-activation cross sections for the target nuclei ${ }^{97} \mathrm{Mo}$ and ${ }^{98} \mathrm{Mo}$. To allow a complete analysis of the systematics, recent earlier results by the KRI $\left({ }^{92} \mathrm{Mo}\right)$ and IRMM ( $\left.{ }^{\text {nat }} \mathrm{Mo}\right)$ groups are shown for three additional reactions, together with the available experimental data for ${ }^{94,95,100}$ Mo.

Comparison with the model calculations are given in all cases, and the calculated contributions of sequential neutron and proton $(\mathrm{np}+\mathrm{pn})$ emission is indicated as $(n, n p)$ in the figures, while the deuteron emission contribution is indicated as $(n, d)$.

For the target nucleus ${ }^{96} \mathrm{Mo}$, three new data points are shown for the cross section of production of the ${ }^{95} \mathrm{Nb}^{\mathrm{m}}$ isomer. These results together with the earlier measurements around $14 \mathrm{MeV}$ establish a smooth excitation curve from 13 to $21 \mathrm{MeV}$. The STAPRE-H model calculation follows the shape suggested by the data but is somewhat too high. For the TALYS calculation, the initial increase in the $(n, d)$ contribution is too sharp and crosses through the data at $14 \mathrm{MeV}$ with a different slope; then the sum of the $(n, n p)$ and $(n, d)$ contributions remains well below the data above $14 \mathrm{MeV}$. Both the STAPRE-H and TALYS calculations show comparable contributions from both the $(n, d)$ and $(n, n p)$ processes above $16 \mathrm{MeV}$, the $(n, d)$ contribution being the larger one throughout. For the total activation cross section on ${ }^{96} \mathrm{Mo}$ for this channel, the only 
available data are from earlier work around $14 \mathrm{MeV}$, and agreement with both TALYS and STAPRE-H is excellent. Again, $(n, n p)$ and $(n, d)$ contributions are of similar importance in both approaches, but contrary to the case of the isomer, the $(n, n p)$ process gives the larger calculated contribution at higher energies. Interesting differences between the two model calculations at higher energy suggest the need for additional measurements for the ${ }^{96} \mathrm{Mo}(n, x){ }^{95} \mathrm{Nb}$ reaction.

The new measurements for the ${ }^{97} \operatorname{Mo}(n, x){ }^{96} \mathrm{Nb}$ cross section are all above $15 \mathrm{MeV}$. No earlier results exist in this energy range, and the new data extrapolated to $14 \mathrm{MeV}$ agree well with the available measurements around that energy. Both STAPRE-H and TALYS yield excitation curves that agree reasonably well with the data, although the STAPRE-H calculation is clearly higher than the measured data above $15 \mathrm{MeV}$. The relative importance of the $(n, n p)$ and $(n, d)$ contributions calculated by the two models is quite different above $15 \mathrm{MeV}$, suggesting the need for emission spectra measurements around $20 \mathrm{MeV}$ to determine the relative weight of these contributions.

For the target nucleus ${ }^{98} \mathrm{Mo}$, two new data points are shown for the total activation cross section of this channel and four for the cross section of the production of the isomer. In both cases, no previously measured data were available for the energy range above $15 \mathrm{MeV}$. The shape of the excitation curve above $13 \mathrm{MeV}$ is now reasonably determined, although for both reactions the new data point near $16 \mathrm{MeV}$ appears somewhat low. The TALYS calculation is in good agreement with all data for the total activation cross section, whereas the STAPRE-H calculation is somewhat high at $18 \mathrm{MeV}$. In the case of the isomer, the STAPRE-H calculation agrees well with the measured data, while the shape suggested by the TALYS calculation appears somewhat low above $16 \mathrm{MeV}$. Differences in the shapes of the $(n, n p)$ and $(n, d)$ contributions between the two calculations partially compensate each other when they are summed.

The earlier results of measurements by the KRI group for the ${ }^{92} \operatorname{Mo}(n, x){ }^{91} \mathrm{Nb}^{\mathrm{m}}$ reaction, which agree well with the results by Qaim et al. [1] obtained via radiochemical separation, thin sample preparation, and X-ray counting [1], are slightly higher than earlier measurements by Liskien et al. [18] and the new model calculations. The agreement with the calculations for $\left.{ }^{92} \mathrm{Mo}(n, x)\right)^{91} \mathrm{Nb}$ is good.

Regarding the recent measurements by the IRMM group for the ${ }^{\text {nat }} \operatorname{Mo}(n, x){ }^{94} \mathrm{Nb}$ reaction [7], the present local approach (STAPRE-H calculation) shows good agreement. The production of ${ }^{94} \mathrm{Nb}$ is seen to be dominated by the contribution from the ${ }^{95} \mathrm{Mo}(n, x){ }^{94} \mathrm{Nb}$ reaction above $14 \mathrm{MeV}$.

Finally, for ${ }^{92,94,95,96} \mathrm{Mo}$, the $(n, d)$ reaction cross section was determined at $14.8 \mathrm{MeV}$ from deuteron-emission spectra measured by Haight et al. [62]. Those data were used to fix the deuteron preformation probability used in the local approach (STAPRE-H calculations) of this work. The values obtained were 0.5 for the target nuclei ${ }^{94,95} \mathrm{Mo}, 0.55$ for ${ }^{96} \mathrm{Mo}$, and 0.6 for ${ }^{92}$ Mo. Similar values of 0.5 and 0.6 were needed to reproduce the $(n, x)$ activation data around the incident energy of $15 \mathrm{MeV}$ for the target nuclei ${ }^{97} \mathrm{Mo}$ and ${ }^{98,100} \mathrm{Mo}$, respectively. These values could be inferred from the measured cross section data since for the heavier Mo isotopes the contribution of the $(n, d)$ reaction dominates (see Figs. 8-9). The good agreement of the
STAPRE-H calculations using the given preformation probabilities with the data of Haight et al. [62] is shown in Figs. 5-7. The TALYS calculations also agree well with the data, while the shape of the excitation function is quite different.

The systematic study of the isotope effect of reaction cross sections at $14.7 \mathrm{MeV}$ [86] proved that the $(n, d)$ and $(n, n p)$ processes are affected by the number of neutrons in the nuclei. The present work shows by means of both the STAPRE-H and TALYS calculations that the contribution of the $(n, d)$ process relative to that of the $(n, n p)$ process, several $\mathrm{MeV}$ above the respective thresholds, grows gradually with mass number. Thus, the $(n, d)$ process is of minor importance for the ${ }^{92}$ Mo compared with the $(n, n p)$ process and dominates entirely for ${ }^{100} \mathrm{Mo}$. The last conclusion was derived also by Qaim [86] by comparison of the experimental data around $14 \mathrm{MeV}$ and the HF statistical model predictions. The calculated ratios of $(n, d)$ and $(n, n p)$ contributions differ greatly between the STAPRE-H and TALYS calculations, suggesting the need for further emission spectra measurements to settle this important issue.

\section{SUMMARY AND CONCLUSIONS}

A large number of new measurements with the activation technique were performed for neutron-induced reactions from 16 to $20 \mathrm{MeV}$ on the stable molybdenum isotopes. Both natural and enriched samples were used, allowing for separation of different reaction channels leading to the same activity. First results were obtained for the ${ }^{92} \mathrm{Mo}(n, 2 n)^{91} \mathrm{Mo}^{\mathrm{m}}$, ${ }^{92} \operatorname{Mo}(n, \alpha){ }^{89} \mathrm{Zr}^{\mathrm{m}},{ }^{94} \mathrm{Mo}(n, 2 n){ }^{93} \mathrm{Mo}^{\mathrm{m}},{ }^{95} \mathrm{Mo}(n, \quad p){ }^{95} \mathrm{Nb}^{\mathrm{m}}$, ${ }^{96} \mathrm{Mo}(n, p){ }^{96} \mathrm{Nb},{ }^{96} \mathrm{Mo}(n, x){ }^{95} \mathrm{Nb}^{\mathrm{m}},{ }^{97} \mathrm{Mo}(n, p){ }^{97} \mathrm{Nb},{ }^{97} \mathrm{Mo}(n$, $\left.\left.p)^{97} \mathrm{Nb}^{\mathrm{m}},{ }^{97} \mathrm{Mo}(n, x){ }^{96} \mathrm{Nb},{ }^{98} \mathrm{Mo}(n, p)\right)^{98} \mathrm{Nb}^{\mathrm{m}},{ }^{98} \mathrm{Mo}(n, x)\right)^{97} \mathrm{Nb}$, $\left.{ }^{98} \mathrm{Mo}(n, x)\right)^{97} \mathrm{Nb}^{\mathrm{m}}$, and ${ }^{100} \mathrm{Mo}(n, \alpha){ }^{97} \mathrm{Zr}$ reactions. A significant body of new good accuracy $14 \mathrm{MeV}$ data has resulted from measurements on natural samples. For cases where the comparison can be made, these new measurements are in excellent agreement with the extrapolated trend of the measurements above $16 \mathrm{MeV}$.

The systematics of $(n, p),(n, \alpha),(n, 2 n)$ and $(\Delta Z=1$, $\Delta A=1)$ reactions for all Mo target isotopes were studied based on the newly measured cross section data as well as all existing measured cross sections below $21 \mathrm{MeV}$. These data were compared with model calculations based on a parameter set determined by global calculations (TALYS code) and, alternatively, based on a parameter set determined by local optimization. Both models treat all involved nuclei and reaction processes in a physically consistent manner.

The global approach leads to remarkably good agreement (within 20-30\%) with the data for total activation cross sections in the case of most $(n, p),(n, 2 n)$ and $(\Delta Z=1$, $\Delta A=1)$ reactions, given the lack of any fine tuning of the parameters involved (blind calculations). On the other hand, the TALYS code, used for these calculations, does not have an optimized $\alpha$ particle optical model, leading often to a poor description of the $(n, \alpha)$ data just above threshold. At higher energies, the calculated $(n, \alpha)$ cross sections obtained in this approach increase too rapidly, suggesting that a revision of the $\alpha$ particle preequilibrium systematics may be 
needed and possibly some of the level density parameters. Furthermore, some deviations are observed for cross sections leading to isomers, since accurate calculations are more sensitive to the level and decay scheme of the nucleus, $\gamma$-ray strength functions, and the detailed level density modeling. In the spirit of blind calculations, these have not been individually studied. The recent improvements in the TALYS code mentioned in Section IIIA have resulted in a better description of $(n, p),(n, d) \ldots(n, \alpha)$ channels and branching ratio data, when compared with the $\mathrm{Ni}$ and $\mathrm{Co}$ study of Ref. [31].

The local approach provides good agreement with the measured data throughout, with only relatively minor deviations for $(\Delta Z=1, \Delta A=1)$ reactions. Agreement with the data just above threshold is evidence for the success of the carefully determined neutron, proton, and $\alpha$ particle optical model parameters. Independent evidence for the quality of the proton OMP was given by comparison with recent data for the ${ }^{93} \mathrm{Nb}(p, n)$ and ${ }^{93} \mathrm{Nb}(p, \gamma)$ reactions below $5.5 \mathrm{MeV}$. Generally, agreement with the data leading to isomers indicates the viability of the adopted level-density model and the $\gamma$-ray strength functions. In particular, the latter almost exclusively determine the very good agreement for the ${ }^{94} \operatorname{Mo}(n, 2 n)^{93} \mathrm{Mo}^{\mathrm{m}}$ reaction. $\gamma$-ray strength functions were adjusted using recent experimental $\gamma$-width values, and their validity was checked by comparison with the available average capture data for all stable Mo isotopes and for ${ }^{93} \mathrm{Nb}$.

From $14 \mathrm{MeV}$ towards higher energies, agreement between model predictions and measured data is increasingly determined by the description of the preequilibrium process. In the local approach, the geometry-dependent hybrid model (GDH), with up-to-date partial level densities, preequilibrium $\alpha$ and deuteron emission, and a first collision Fermi energy determined by the local density approach, describes the data in this energy range very well.

For the $(n, \alpha)$ reactions, the broad experimental excitation curves are well described by the STAPRE-H calculation featuring PE $\alpha$ emission in the GDH model. Thus, the viability is demonstrated of both the level-density model and the approach taken to $\alpha$-particle preequilibrium emission in the present local approach. An $\alpha$-particle preformation probability of 0.18 was used. It was determined by comparison with measured emission spectra at 14.7 MeV.
For the $(n, d)$ reaction, $\mathrm{PE}$ emission was modeled with STAPRE-H in the same way as for the $\alpha$ particles with deuteron preformation probabilities in the range of $0.5-0.6$, as determined from measured emission spectra. Comparisons show that both calculations give comparable agreement with the measured cross section data. However, the relative contributions of the deuteron emission and the sequential neutronproton emission processes are widely different between the models with pronounced differences in energy dependence. To resolve this interesting matter would require additional measurements of charged-particle emission spectra at an energy around $20 \mathrm{MeV}$.

In general, the database and the model description for the neutron-induced reactions on the Mo isotopes are now rather well established. On the other hand, for some reactions, such as the ${ }^{95} \mathrm{Mo}(n, x){ }^{94} \mathrm{Nb}$ and ${ }^{96} \mathrm{Mo}(n, x){ }^{95} \mathrm{Nb}$ reactions, large differences are observed between the model calculations that would require additional measurements to resolve the origin(s) of the discrepancies. For the important ${ }^{94} \operatorname{Mo}(n, 2 n)^{93} \mathrm{Mo}$ reaction, both models agree but deviate greatly from the only two available data points, suggesting again the need for further measurements to clarify this matter. Finally, comparison of the two model predictions for the ${ }^{94} \mathrm{Mo}(n, p){ }^{94} \mathrm{Nb}$ and ${ }^{94} \mathrm{Mo}(n, p){ }^{94} \mathrm{Nb}^{\mathrm{m}}$ reactions away from $14 \mathrm{MeV}$ demonstrates that blind model predictions that generally perform very well may still show deviations that exceed $30 \%$. Thus, it is evident that further improvement of nuclear modeling in the interest of the study of underlying mechanisms and applications is desirable and requires new, high quality data.

\section{ACKNOWLEDGMENTS}

P.R. is grateful to the Commission of the European Communities for a fellowship. V.A. is grateful to the EC/JRC for support to carry out research at the IRMM Van de Graaff laboratory during September-October 2000. A.F. is grateful to UKAEA for support of experiments. This work was supported in part by the EC/JRC/IRMM, the Contract QS0480 between UKAEA and KRI, and the Contract of Association between EURATOM and MEC-Bucharest No. ERB-5005-CT-990101. The present authors thank Drs. Y. Ikeda and Y. Kasugai for making available the enriched samples that made a large part of this work possible.
[1] S. M. Qaim, R. Wölfle, and B. Strohmaier, Phys. Rev. C 40, 1993 (1989).

[2] P. E. Garrett, L. A. Bernstein, J. A. Becker, K. Hauschild, C. A. McGrath, D. P. McNabb, W. Younes, M. B. Chadwick et al., Phys. Rev. C 62, 054608 (2000).

[3] N. Yamamuro, Nucl. Sci. Eng. 109, 128 (1991).

[4] A. Fessler, E. Wattecamps, D. L. Smith, and S. M. Qaim, Phys. Rev. C 58, 996 (1998).

[5] A. Fessler, A. J. M. Plompen, D. L. Smith, and Y. Ikeda, Nucl. Sci. Eng. 134, 171 (2000).

[6] P. Reimer, V. Avrigeanu, A. J. M. Plompen, and S. M. Qaim, Phys. Rev. C 65, 014604 (2001).
[7] P. Reimer, M. Hult, A. J. M. Plompen, P. N. Johnston, V. Avrigeanu, and S. M. Qaim, Nucl. Phys. A705, 265 (2002).

[8] M. B. Chadwick et al., Nucl. Sci. Eng. 131, 293 (1999).

[9] A. Koning et al., J. Nucl. Sci. Technol. Suppl. 2, 1161 (2002).

[10] A. J. M. Plompen, D. L. Smith, P. Reimer, S. M. Qaim, V. Semkova, F. Cserpak, V. Avrigeanu, and S. Sudar, J. Nucl. Sci. Technol. Suppl. 2, 192 (2002).

[11] A. A. Filatenkov and S. V. Chuvaev, V. G. Khlopin Radium Institute Report RI-252 (in Russian), 1999 (unpublished).

[12] A. A. Filatenkov and S. V. Chuvaev, V. G. Khlopin Radium Institute Report KRI-258, 2001 (unpublished). 
[13] M. Avrigeanu, V. Avrigeanu, and A. J. M. Plompen, J. Nucl.break; Sci. Technol. Suppl. 2, 803 (2002); V. Avrigeanu, in Proceedings of the Workshop of the OECD-NEA WPEC Subgroup 19 on Activation Cross Sections, Geel, 2003, edited by A. J. M. Plompen (in press), and http://tandem.nipne.ro/vavrig/ 2003/Conferences/WWPECVA.DOC.

[14] K. J. R. Rosman and P. D. P. Taylor, Pure Appl. Chem. 70, 217 (1998).

[15] A. A. Filatenkov, S. V. Chuvaev, V. A. Jakovlev, and V. P. Popik, Fusion Eng. Design 37, 151 (1997); A. A. Filatenkov, S. V. Chuvaev, V. N. Aksenov, and V. A. Jakovlev, International Atomic Energy Agency (IAEA) Report INDC(CCP)-402, Vienna, 1997 (unpublished).

[16] V. Avrigeanu, S. Sudár, Cs. M. Buczkó, J. Csikai, A. A. Filatenkov, S. V. Chuvaev, R. Dóczy, V. Semkova, and V. A. Zelenetsky, Phys. Rev. C 60, 017602 (1999).

[17] I. G. Birn (private communication).

[18] H. Liskien, R. Wölfle, R. Widera, and S. M. Qaim, Appl. Rad. Isotop. 41, 83 (1990).

[19] H. H. Anderson and J. F. Ziegler, Hydrogen Stopping Powers and Ranges in All Elements (Pergamon, New York, 1977).

[20] G. Lövestam (private communication).

[21] M. Drosg, Report IAEA-NDS-87, IAEA, Vienna, 2000 (unpublished), www-nds.iaea.org/drosg2000.html.

[22] J. Ziegler (unpublished), www.srim.org.

[23] Nuclear Data Standards for Nuclear Measurements, edited by H. Condé (NEA Nuclear Data Committee, Paris, 1992).

[24] A. J. M. Plompen, BgNS Trans. 9, 131 (2004).

[25] B. Jäckel, W. Westmeier, and P. Patzelt, Nucl. Instrum. Methods Phys. Res. A 261, 543 (1987).

[26] Gamma- and X-Ray Spectrometry with Semiconductor Detectors, edited by K. Debertin and R. G. Helmer (Elsevier, North Holland, Amsterdam, 1988).

[27] C. L. Dunford and R. R. Kinsey, IAEA-NDS-205 (BNL-NCS65687), IAEA, Vienna, 1998 (unpublished).

[28] R. B. Firestone, Table of Isotopes, 8th ed. (Wiley, New York, 1995).

[29] M. Wagner, H. Vonach, A. Pavlik, B. Strohmaier, S. Tagesen, and J. Martinez-Rico, Phys. Data 13-5 (1990).

[30] A. J. Koning, S. Hilaire, and M. C. Duijvestijn, in Proceedings of the International Conference on Nuclear Data for Science and Technology, Santa Fe, NM, 26 Sep-1 Oct (2004), (to be published).

[31] V. Semkova, V. Avrigeanu, T. Glodariu, A. J. Koning, A. J. M. Plompen, D. L. Smith, and S. Sudár, Nucl. Phys. A730, 255 (2004).

[32] P. D. Kunz, DWUCK4 User Manual (OECD/NEA Data Bank, 1984).

[33] M. Avrigeanu, M. Ivascu, and V. Avrigeanu, Z. Phys. A 335, 299 (1990); M. Avrigeanu and V. Avrigeanu, Institute of Physics and Nuclear Engineering Report NP-86-1995, Bucharest, 1995 (unpublished).

[34] A. J. Koning and J. P. Delaroche, Nucl. Phys. A713, 231 (2003).

[35] S. Watanabe, Nucl. Phys. 8, 484 (1958).

[36] J. Raynal, Technical Report CEA-N-2772, CEA, Saclay, France, 1994 (unpublished).

[37] C. Kalbach, Phys. Rev. C 33, 818 (1986).

[38] IAEA-NDS, www-nds.iaea.or.at, RIPL-2, IAEA, 1400 Vienna, 2003.

[39] A. V. Ignatyuk, Sov. J. Nucl. Phys. 21, 255 (1975).
[40] A. J. Koning and M. C. Duijvestijn, Nucl. Phys. A744, 15 (2004).

[41] S. F. Mughabghab, M. Divadeenam, and N. E. Holden, Neutron Cross Sections (Academic Press, Orlando, FL, 1981).

[42] V. Avrigeanu, T. Glodariu, A. J. M. Plompen, and H. Weigmann, J. Nucl. Sci. Technol. Suppl. 2, 746 (2002).

[43] W. Dilg, W. Schantl, H. Vonach, and M. Uhl, Nucl. Phys. A217, 269 (1973).

[44] Report IAEA-TECDOC-1034, Vienna, 1998; http://iaeand.iaea. or.at/ripl (unpublished).

[45] J. P. Delaroche, Ch. Lagrange, and J. Salvy, in Nuclear Theory in Neutron Nuclear Data Evaluation, Report IAEA-190 (IAEA, Vienna, 1976), vol. 1, p. 251.

[46] "EXFOR-EXchange FORmat for nuclear data," National Nuclear Data Center, Brookhaven National Laboratory, www.nndc.bnl.gov/nndc/exfor.

[47] Ch. Lagrange, in Neutron Data for Structural Materials for Fast Reactors, edited by K. H. Bockhoff (Pergamon, Oxford, 1979), p. 756.

[48] A. B. Smith, J. Phys. G 26, 1467 (2000).

[49] M. Ivascu, M. Avrigeanu, and V. Avrigeanu, Institute for Physics and Nuclear Engineering Report NP-28-1983, Bucharest, 1983 (unpublished).

[50] C. Kalbach, Phys. Rev. C 62, 044608 (2000).

[51] C. H. Johnson, A. Galonsky, and R. L. Kernell, Phys. Rev. C 20, 2052 (1979).

[52] D. S. Flynn, R. L. Hershberger, and F. Gabbard, Phys. Rev. C 20, 1700 (1979); R. Schrils, D. S. Flynn, R. L. Hershberger, and F. Gabbard, ibid. 20, 1706 (1979); R. L. Hershberger, D. S. Flynn, F. Gabbard, and C. H. Johnson, ibid. 21, 896 (1980); C. H. Johnson, ibid. 22, 899 (1980).

[53] S. M. Grimes, Phys. Rev. C 22, 436 (1980).

[54] Ch. Lagrange, Phys. Rev. C 22, 896 (1980).

[55] F. G. Perey, Phys. Rev. 131, 745 (1963).

[56] S. Harissopulos, E. Skreti, P. Tsagari, G. Souliotis, P. Demetriou, T. Paradellis, J. W. Hammer, R. Kunz, C. Angulo, S. Goriely, and T. Rauscher, Phys. Rev. C 64, 055804 (2001).

[57] D. Wilmore and P. E. Hodgson, Nucl. Phys. 55, 673 (1964).

[58] F. D. Becchetti and G. W. Greenlees, Phys. Rev. 182, 1190 (1969).

[59] C. H. Johnson, Phys. Rev. C 16, 2238 (1977).

[60] V. Avrigeanu, P. E. Hodgson, and M. Avrigeanu, Phys. Rev. C 49, 2136 (1994).

[61] J. M. Lohr and W. Haeberli, Nucl. Phys. A232, 381 (1974).

[62] R. C. Haight, S. M. Grimes, R. G. Johnson, and H. H. Barschall, Phys. Rev. C 23, 700 (1981).

[63] D. G. Gardner and F. S. Dietrich, Lawrence Livermore National Laboratory Report UCRL-82998, 1979 (unpublished).

[64] M. Avrigeanu, V. Avrigeanu, G. Căta, and M. Ivascu, Rev. Roum. Phys. 32, 837 (1987).

[65] G. de Saussure, N. M. Larson, J. A. Harvey, and N. W. Hill, Ann. Nucl. Energy 19, 393 (1992); F. Corvi, M. C. Moxon, and $\mathrm{K}$. Athanassopulos, in Proceedings of the International Conference on Nuclear Data for Science and Technology, Gatlinburg, May 1994, edited by J. K. Dickens (American Nuclear Society, La Grange Park, 1994) p. 221; F. Corvi, G. Fioni, F. Gunsing, P. Mutti, and L. Zanini, Nucl. Phys. A697, 581 (2002).

[66] A. J. M. Plompen, V. Avrigeanu, C. Borcea, L. Olah, and V. Semkova, in Proceedings of the International Conference PHYSOR-2002, Seoul, 2002 (unpublished).

[67] M. Blann, Phys. Rev. Lett. 28, 757 (1972); Nucl. Phys. A213, 
570 (1973); M. Blann and H. K. Vonach, Phys. Rev. C 28, 1475 (1983).

[68] M. Avrigeanu and V. Avrigeanu, Comp. Phys. Commun. 112, 191 (1998); A. Harangozo, I. Stetcu, M. Avrigeanu, and V. Avrigeanu, Phys. Rev. C 58, 295 (1998).

[69] C. Kalbach, Phys. Rev. C 32, 1157 (1985).

[70] M. Avrigeanu and V. Avrigeanu, J. Phys. G 20, 613 (1994).

[71] M. Avrigeanu, A. Harangozo, V. Avrigeanu, and A. N. Antonov, Phys. Rev. C 54, 2538 (1996).

[72] E. Gadioli and E. Gadioli-Erba, Z. Phys. A 299, 1 (1981).

[73] E. Gadioli, E. Gadioli-Erba, J. J. Hogan, and K. I. Burns, Z. Phys. A 301, 289 (1981).

[74] M. M. Rahman and S. M. Qaim, Nucl. Phys. A435, 43 (1985).

[75] C. Kalbach, Users Manual for PRECO-2000: Exciton Model Pre-Equilibrium Code with Direct Reactions (Duke University, Durham, NC, 2001).

[76] A. Marcinkowski, K. Stakiewicz, U. Garuska, and M. Herman, Z. Phys. A 323, 91 (1986).

[77] R. Doczi, V. Semkova, A. Fenyvesi, N. Yamamuro, Cs. M. Buczko, and J. Csikai, Nucl. Sci. Eng. 129, 164 (1998).

[78] N. I. Molla, R. U. Miah, S. Basunia, S. M. Hossain, and
M. M. Rahman, in Proceedings of the International Conference on Nuclear Data for Science and Technology, Trieste, Italy, 19-24 May, 517 (1997); N. I. Molla, M. M. Rahman, S. Khatun, A. K. M. Fazlul Hoque, R. U. Miah, and A. A. Khan, International Atomic Energy Agency (IAEA) Report INDC(BAN)-003, 1986 (unpublished).

[79] X. Kong, Y. Wang, J. Yuan, X. Wang, J. Yang, and J. Wang, J. High Energy Phys. and Nucl. Phys., 15, 549 (1991).

[80] A. Abboud, P. Decowski, W. Grochulski, A. Marcinkowski, J. Piotrowski, K. Siwek, and Z. Wilhelmi, Nucl. Phys. A139, 42 (1969).

[81] M. Bormann, H.-K. Feddersen, H.-H. Holscher, W. Scobel, and H. Wagner, Z. Phys. A 277, 203 (1976).

[82] J. E. Brolley, Jr., J. L. Fowler, and L. K. Schlacks, Phys. Rev. 88, 618 (1952).

[83] L. R. Greenwood, International Atomic Energy Agency (IAEA) Report IAEA-TECDOC-572, 45, 1989 (unpublished).

[84] N. I. Molla and S. M. Qaim, Nucl. Phys. A283, 269 (1977).

[85] M. Avrigeanu and V. Avrigeanu, in Proceedings of the International Conference on Nuclear Data for Science and Technology, Jülich, 1991, edited by S. M. Qaim (Springer-Verlag, Berlin, 1992), p. 993.

[86] S. M. Qaim, Nucl. Phys. A382, 255 (1982). 